\title{
Comparative transcriptome analysis reveals defense responses against soft rot in Chinese cabbage
}

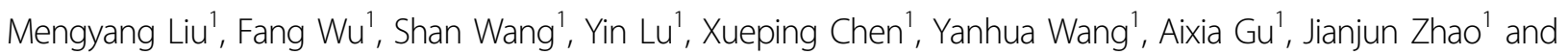
Shuxing Shen ${ }^{1}$

\begin{abstract}
Pectobacterium carotovorum ssp. carotovorum (PCc) is a necrotrophic bacterial species that causes soft rot disease in Chinese cabbage. In this study, plants harboring the resistant mutant $s r$ gene, which confers resistance against $P c c$, were screened from an $800 \mathrm{M}_{2}$ population mutated by ethyl methane sulfonate (EMS) and scored in vitro and in vivo for lesion size. The transcript profiles showed $\sim 512$ differentially expressed genes (DEGs) between sr and WT plants occurring between 6 and $12 \mathrm{~h}$ postinoculation (hpi), which corresponded to the important defense regulation period (resistance) to PCC in Chinese cabbage. The downstream defense genes (CPK, CML, RBOH MPK3, and MPK4) of pathogen pattern-triggered immunity (PTI) were strongly activated during infection at $12 \mathrm{hpi}$ in resistant mutant sr; PTI appears to be central to plant defense against PCC via recognition by three putative pattern recognition receptors (PRRs; BrLYM1-BrCERK1, BrBKK1/SERK4-PEPR1, BrWAKS). Pcc triggered the upregulation of the jasmonic acid (JA) and ethylene (ET) biosynthesis genes in mutant $s r$, but auxins and other hormones may have affected some negative signals. Endogenous hormones (auxins, JAs, and SA), as well as exogenous auxins (MEJA and BTH), were also verified as functioning in the immune system. Concurrently, the expression of glucosinolate and lignin biosynthesis genes was increased at $12 \mathrm{hpi}$ in resistant mutant $s \mathrm{r}$, and the accumulation of glucosinolate and lignin also indicated that these genes have a functional defensive role against $P$ cc. Our study provides valuable information and elucidates the resistance mechanism of Chinese cabbage against Pcc infection.
\end{abstract}

\section{Introduction}

Chinese cabbage (Brassica rapa ssp. pekinensis) originated in central China and is the most widely grown, important vegetable crop in Asia. Soft rot disease caused by the pathogen Pectobacterium carotovorum ssp. carotovorum (Pcc), also known as Erwinia carotovora ssp. carotovora $(E c c)$, can result in severe losses and is one of the three most economically important diseases of Chinese cabbage. The narrow genetic background of the core collections of Chinese cabbage and the little information

\footnotetext{
Correspondence: Aixia Gu (aixiagu@126.com) or Jianjun Zhao (jjz1971@aliyun. com) or Shuxing Shen (shensx@hebau.edu.cn)

'Key Laboratory of Vegetable Germplasm Innovation and Utilization of Hebei, Collaborative Innovation Center of Vegetable Industry in Hebei, College of Horticulture, Hebei Agricultural University, 071000 Baoding, China
}

available about the molecular mechanism of resistance against $P c c$ have resulted in very limited breeding material exhibiting resistance to the disease. $P c c$ is a necrotrophic bacterium with a wide host range ${ }^{1}$ and can survive in the soil for several months without the host. It infects the host through natural pores on the plant surface or wounds, and when environmental conditions such as moisture, oxygen, and temperature are conducive, it exists in the vascular tissue, including parenchyma cells ${ }^{2,3}$.

When Pcc invades the host plant, plant cell walldegrading enzymes (PCWDEs) such as polygalacturonase (PGs), pectate lyase (Pel), and cellulase (Cel) are synthesized and secreted from the bacterial cytosol into intercellular spaces of the plant tissue ${ }^{4}$. Pcc employs the Type II secretion system (T2SS), which is the main way that

\section{(c) The Author(s) 2019}

(c) (i) Open Access This article is licensed under a Creative Commons Attribution 4.0 International License, which permits use, sharing, adaptation, distribution and reproduction in any medium or format, as long as you give appropriate credit to the original author(s) and the source, provide a link to the Creative Commons license, and indicate if changes were made. The images or other third party material in this article are included in the article's Creative Commons license, unless indicated otherwise in a credit line to the material. If material is not included in the article's Creative Commons license and your intended use is not permitted by statutory regulation or exceeds the permitted use, you will need to obtain permission directly from the copyright holder. To view a copy of this license, visit http://creativecommons.org/licenses/by/4.0/. 
proteins are delivered to host cells and cause soft rot disease $^{5,6}$. The type III secretion system (T3SS) has significant roles by contributing to virulence in hemibiotrophic phytopathogenic bacteria for secreting effectors and transporting virulence factors, but few factors are required for $P c c$ to attack the host plant. Except for $D s p E$, no T3SS effectors have been identified that elicit plant cell death to promote plant tissue maceration but not to suppress basal defense responses ${ }^{7,8}$. Therefore, the pathogenicity of $P c c$ does not rely on T3SS to infect host plants 9 .

No resistance genes ( $R$ genes) have been identified for Pectobacterium; these genes encode the proteins that can directly and indirectly recognize effectors and elicit defensive reactions against effectors ${ }^{10}$. The R-genemediated immune response, also known as effectortriggered immunity $(\mathrm{ETI})^{11}$, is pathogen specific; intense, inducing programmed cell death (PCD); and causes the hypersensitive response (HR) so that pathogens cannot obtain nutrition from infection plant parts ${ }^{12}$. Except for ETI in the host-pathogen interaction system, the plant's pattern recognition receptors (PRRs) on the surface of cell membranes recognize conserved microbe- or pathogen-associated molecular patterns (MAMPs/ PAMPs). Pathogen pattern-triggered immunity (PTI $)^{11}$ is a consequence, and recognition of the pathogen causes a series of host responses, which include eliciting production of reactive oxygen species (ROS), activating the $\mathrm{Ca}^{2+}$-mediated and hypersensitive responses, and stimulating the mitogen-activated protein kinase (MAPK) cascade reaction. In addition, the molecular fragments from degradation of the cell wall can act as dangerassociated molecular patterns (DAMPs) and are recognized by PRRs to activate $\mathrm{PTI}^{13}$. Specifically, plant cell wall fragments released by the action of the hydrolytic enzymes secreted by $P c c$ are major elicitors in enhanced immunity toward these pathogens ${ }^{14}$.

Plant hormones have an important role in the regulation of plant growth and development, and they mediate defense responses as signals to pathogens and phytophagous insects ${ }^{15}$. Salicylic acid (SA), jasmonic acid (JA), and ethylene (ET) are primary signals that activate and facilitate immune responses in plants ${ }^{16}$. SA signaling commonly regulates plant defense against biotrophic pathogens, and JA/ET-dependent signaling pathways are required for resistance to necrotrophic pathogens ${ }^{17}$. JA/ ET-dependent signaling pathways have an essential role in resistance to $P c c$, but it is unknown whether the SAdependent pathway is required for plant resistance ${ }^{18-20}$. Additionally, other hormones, such as auxins, abscisic acid (ABA), gibberellins and cytokinins, are considered modulators of plant-pathogen interactions ${ }^{21}$.

WRKYs are one of the largest families of plant transcription factors, with the conserved WRKY domain regulating plant responses to pathogens. WRKY7O is the key factor in balancing SA-dependent signaling and JAdependent signaling for defense against $P c c^{20}$. WRKY 75 positively regulates JA- or SA-dependent defense ${ }^{22}$, and WRKY33 is a positive regulator of JA-dependent genes but represses the SA-dependent pathway ${ }^{23,24}$. In Arabidopsis, WRKY7 activated the expression of the JA-dependent signaling gene PDF1.2, indicating that WRKY7 is a positive regulatory factor in the JA pathway ${ }^{25}$. Overexpression of encoding pineapple bromelain (BAA1), rice leucine-rich repeat-protein (OsLRP) and polygalacturonase-inhibiting protein 2 (PGIP2) ${ }^{26-28}$ was reported to improve resistance to $P c c$ infection.

Plant resistance to $P c c$ is complex, and little is known about the molecular basis of resistance to this soft rot phytopathogen. The completely sequenced $B$. rapa genome furnishes exceptional amounts of genetic data ${ }^{29}$ that can be used for mutant library research in Chinese cabbage. In our previous research, 5396 mutant plants $\left(\mathrm{M}_{1}\right)$ were obtained from seeds after treatment with ethyl methane sulfonate (EMS) mutagenesis. All plants were self-pollinated, and 4253 plants produced between 10 and 300 seeds each, which represented the mutant population $^{30,31}$. RNA-Seq is a transcriptome analysis approach using deep-sequencing technology and has replaced previous technologies such as microarrays ${ }^{32}$. RNA-Seq is a more robust method to reveal global gene expression patterns of plant immunity in response to wild-type (WT) and resistant mutant $s r$ soft rot bacterial infection over time. Therefore, the specific objectives in our research were as follows:

1. to create a reliable identification inoculation method for Pcc and obtain resistant mutants against soft rot from our population mutated by EMS;

2. to determine the seminal period corresponding to defense regulation (resistance) to Pcc;

3. to compare the transcript profiles of resistant mutant sr plants to the susceptible WT plants at 0,6 , 12 and 24 hpi (hours postinoculation) in response to Pcc using RNA-Seq to elucidate the putative resistance molecular mechanism operating against Pcc, including the infection process and recognition of the pathogen, signal transduction and synthesized secondary metabolites functioning in the immune system.

\section{Material and methods}

\section{Plant materials and bacterial pathogen inoculation}

The soft rot-resistant mutant $s r$ was screened from an EMS-mutagenized $\mathrm{M}_{2}$ population of Chinese cabbage ${ }^{30,31}$ and controlled self-pollinated to obtain $\mathrm{M}_{4}$ generation. All seeds were sown in pots in the greenhouse at $26-28{ }^{\circ} \mathrm{C}$ with $16 \mathrm{~h}$ daytime $/ 15{ }^{\circ} \mathrm{C}$ with $8 \mathrm{~h}$ nighttime and $90 \%$ humidity. All samples were collected one week after transplanting. 
Pcc pathogen $B C 1^{33}$ was cultured in LB broth medium overnight in an incubator set at $28{ }^{\circ} \mathrm{C}$ with continuous shaking $(150 \mathrm{rpm})$. Bacteria were diluted with LB medium to $10^{5} \mathrm{cfu} / \mathrm{mL}$ for inoculation of plants.

Petioles of the third leaves (from inside to outside) of 7-to-8 leaf plants were lightly scored (through the epidermis) with a sterile scalpel and inoculated with 5-10 $\mu \mathrm{L}$ of a uniform bacterium suspension made from cultures, which were labeled "in vivo"34 (Supplementary Fig. 1a). Similarly, the third leaves were cut into 5.5 -cm-diameter disks with a homemade tool (Supplementary Fig. 1) and placed in closed 9-cm-diam petri dishes with two layers of moist filter paper to maintain high humidity. The leaf circles were scored as before, inoculated with $5-10 \mu \mathrm{L}$ of bacterium suspension, and placed in an incubator $\left(28^{\circ} \mathrm{C}\right.$, $90 \%$ humidity). These cultures were designated as "in vitro" 28 (Supplementary Fig. 1b).

\section{Harvesting samples and observing disease severity}

For RNA-Seq analyses, the leaves that were to be inoculated with WT and $s r$ lines in vivo were harvested 0 hpi (control) and after inoculation (6,12 and $24 \mathrm{hpi}$ ) with three biological replicates. Samples at 0 and $12 \mathrm{hpi}$ in WT and $s r$ were used to determine the concentrations of glucosinolate, lignin and hormones. All samples were frozen immediately in liquid nitrogen and stored at $-80{ }^{\circ} \mathrm{C}$ before analyses.

To accurately evaluate the visible symptoms of $P c c$, leaves were inoculated in vivo and in vitro. Disease severity in vivo was scored at $48 \mathrm{hpi}$ because of lower humidity and subsequent disease development compared to in vitro trials. Disease ratings are illustrated in Fig. 1a: 0 (no symptoms), 1 (lesions discrete and $<0.5 \mathrm{~cm}$ in diam, lignified inoculation spots), 3 (lesions discrete and $0.5-2 \mathrm{~cm}$ in diam, lignified inoculation spots), 5 (macerated lesions occupied less than $60 \%$ of the petiole), 7 (macerated lesions occupied more than $60 \%$ of the petiole), and 9 (macerated lesions occupied the entire petiole and extended to the leaf blade). Plants with disease severity scores of 0,1 , and 3 were categorized as resistant ( 0 was fully resistant, whereas 1 and 3 were partially resistant); scores of 5 were categorized as partially susceptible; and scores of between 7 and 9 were categorized as susceptible. Lesion diameters on in vitro plants were measured using ImageJ software (National Institutes of Health, USA). The macerated lesions on the leaf disks were scored based on a modification of Park et al. $^{28}$ at 24 hpi as disease severity (Fig. 1b): 0 (no symptoms), 1 (discrete lesions $<0.5 \mathrm{~cm}$ in diam: lignified inoculation spots), 3 (discrete lesions $0.5-1.5 \mathrm{~cm}$ in

$\mathbf{a}$
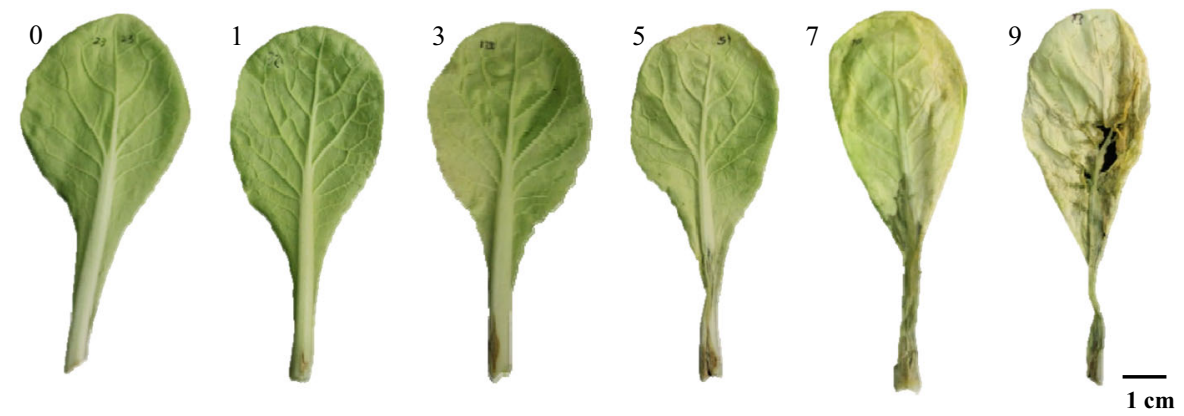

b
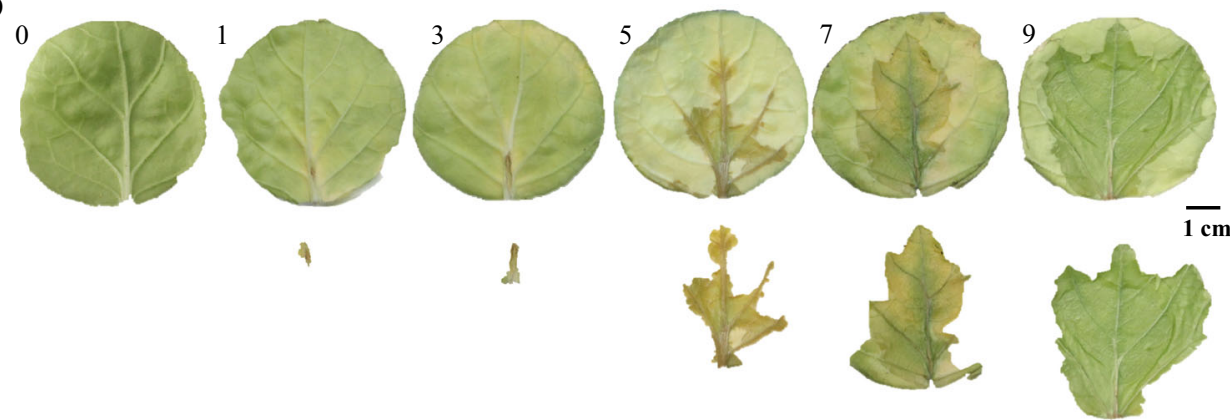

Fig. 1 The disease grading standard $(0,1,3,5,7,9)$ for soft rot resistance of Chinese cabbage seedlings caused by Pcc. a The macerated lesions on the leaves were cored in vivo. $\mathbf{b}$ The macerated lesions on the leaves were cored in vitro 
diameter, lignified inoculation spots), 5 (macerated lesions occupied $25-35 \%$ of the entire leaf disk), 7 (macerated lesions occupied $35-50 \%$ of the entire leaf disk), and 9 (macerated lesions occupied more than 50\% of the entire leaf disk).

\section{cDNA library construction and sequencing data analysis}

The RNA from three biological replicates of each mutant $s r$ and WT from 0, 6, 12, and 24 hpi was extracted according to the manufacturer's instructions using Trizol reagent (Invitrogen, USA). RNA purity was assessed, and the cDNA library was prepared as previously described ${ }^{32}$.

Raw data (raw reads) in the fastq format were processed and cleaned (clean reads). The clean data were mapped to the $B$. rapa reference genome (v1.5) from the Brassica database (BRAD) (http://brassicadb.org/brad//) ${ }^{29}$. After filtering the reads, $179.17 \mathrm{~Gb}$ of high-quality sequences (more than $96 \%$ of the raw reads) of 24 samples (WT and $s r$ at $0,6,12,24$ hpi with three replicates) were obtained, ranging from 6.16 to $9.16 \mathrm{~Gb}$ per sample, with error rates $<0.1 \%$ and $67.60-75.31 \%$; $66.71-74.36 \%$ of these sequences were mapped to unique locations, whereas $0.89-1.55 \%$ were mapped to multiple genome locations (Supplementary Table S1). A total of 44248 predicted B. rapa genes were annotated.

HTSeq v0.6.1 was used to count the read numbers mapped to each gene, and the FPKM (Fragments Per Kilobase of transcript sequence per Million base pairs sequenced) of each gene was calculated based on the length of the gene and read counts mapped to this gene $\mathrm{e}^{35}$. Differential expression analyses of two groups were performed using the DESeq R package (1.18.0). The resulting $P$-values were adjusted to control the false discovery rate (FDR). Genes with an adjusted $P$-value $\leq 0.05$ found by DESeq were considered differentially expressed genes (DEGs). We used KOBAS software to test the statistical enrichment of DEGs in KEGG pathways.

\section{Quantitative real-time PCR (qRT-PCR) analyses}

Total RNA was extracted from the same plant samples as those used for RNA-Seq, and first-strand cDNA was synthesized using a ReverTra Ace qPCR RT Master Mix (TOYOBO, Japan) according to the manufacturer's instructions. Bractin was used as an internal reference control, and gene primers were designed by Primer Premier 5.0 software. qRT-PCR analysis was performed on a Lightcycler 96 real-time PCR detection system (Roche, USA) using THUNDERBIRD SYBR qPCR Mix as a fluorescent detection dye (TOYOBO, Japan). The qRTPCR program was performed in 96-well plates under the following protocol: initial activation at $95{ }^{\circ} \mathrm{C}$ for $10 \mathrm{~min}$, followed by 45 cycles of $95^{\circ} \mathrm{C}$ for $10 \mathrm{~s}, 58^{\circ} \mathrm{C}$ for $10 \mathrm{~s}$, and $68{ }^{\circ} \mathrm{C}$ for $10 \mathrm{~s}$. This procedure was followed by melting curve analysis from $95^{\circ} \mathrm{C}$ for $10 \mathrm{~s}, 65^{\circ} \mathrm{C}$ for $60 \mathrm{~s}$, and $97^{\circ} \mathrm{C}$ for $1 \mathrm{~s}$. The $2^{-\triangle \triangle \mathrm{Ct}}$ method was used to calculate the relative expression levels of the target genes ${ }^{36}$. All reactions were performed with three biological and technical replicates.

\section{Glucosinolate determination}

Glucosinolate was extracted according to the method described by Liao et $\mathrm{al}^{37}$, and compounds were detected using $\mathrm{HPLC}^{38}$. Each sample was analyzed with three biological replicates.

\section{Lignin content determination}

Lignin was extracted according to the method described by Johnson et al. ${ }^{39}$. Three biological replicates of each of the mutant $s r$ and WT at 0 and $12 \mathrm{hpi}$ were freeze dried and ground into powder. Samples $(1.5 \mathrm{mg}$ of DW (dry weight)) were added to $1.5 \mathrm{~mL}$ of $20-40 \%$ acetyl bromide and $0.2 \mathrm{~mL}$ of perchloric acid and maintained at $70^{\circ} \mathrm{C}$ for $1 \mathrm{~h}$. Afterward, $3 \mathrm{~mL}$ of $2 \mathrm{M}$ $\mathrm{NaOH}$ and $3 \mathrm{~mL}$ of glacial acetic acid were added, and then the entire reaction was diluted to $25 \mathrm{~mL}$ with $100 \%$ glacial acetic acid. The absorbance of the reactions was measured at $280 \mathrm{~nm}$ with a UV-1800 spectrophotometer (Shimadzu, Japan), and the mean amount of lignin was calculated for each sample from five biological replicates.

\section{IAAs, JAs, and SA determination using LC-MS/MS}

Fresh leaves from mutant $s r$ and WT were harvested at 0 and $12 \mathrm{hpi}$, weighed, immediately frozen in liquid nitrogen, and stored at $-80^{\circ} \mathrm{C}$. Sample extracts were analyzed using an LC-ESI-MS/MS system (HPLC, Shimpack UFLC SHIMADZU CBM30A system, www. shimadzu.com.cn/; MS, Applied Biosystems 6500 Triple Quadrupole) and an API 6500 QTRAP LC/MS/MS system (AB Sciex, USA) ${ }^{40}$.

\section{Hormone treatment in vitro}

The third leaves of WT and $s r$ plants were harvested at the same time that in vitro inoculations were completed. Additionally, samples from soft rot-tolerant pak choi ('Huaguan') were collected. Aqueous solutions of the phytohormones (IAA $(200 \mu \mathrm{M})$, IBA $(200 \mu \mathrm{M})$, Me-JA $(1 \mathrm{mM})$, and BTH $\left.(0.1 \mathrm{mM})^{41,42}\right)$ were sprayed onto plants, which were wrapped with a layer of plastic film for $12 \mathrm{~h}$. The film was removed, and the plants were inoculated with $P c c$ via the previously described protocol for in vitro studies. Controls were treated with sterile, distilled water. After the plants had 7-8 leaves, the petioles of the third leaf (from inside to outside) were wounded, inoculated with $5-10 \mu \mathrm{L}$ of fresh bacterial suspension as before and identified as in vivo ${ }^{34}$ (Supplementary Fig. 1a). Three biological replicates for $s r$ and WT inoculations were made. 


\section{Results}

Screening the mutants resistant to Pcc and scoring the disease severity in the $M_{2}$ population

We randomly chose $800 \mathrm{M}_{2}$ plants from 400 different $M_{1}$ families to be inoculated with Pcc for in vitro and in vivo studies of Chinese cabbage (Supplementary Table 2). Disease severity was observed at $24 \mathrm{hpi}$ in vitro ${ }^{28}$ and at $48 \mathrm{hpi}$ in vivo ${ }^{34}$ (Supplementary Fig. 1). In the $\mathrm{M}_{2}$ population, the greatest disease grade was 9, and most plants were susceptible to Pcc. The disease severity of WT plants inoculated with $P c c$ was scored as 9 by both inoculation methods, and all were susceptible to Pcc (Fig. 2a). Only one plant from the $\mathrm{M}_{2}$ population was evaluated as resistant (disease grade 1) in both in vivo and in vitro methods and thereafter was referred to as $s r$ (Fig. 2b). After 7 days of inoculation with $P c c$, the resistant mutant $s r$ plants were still alive; in contrast, WT plants were dead (Supplementary Fig. 2).

\section{Differentially expressed genes (DEGs) between WT and sr at four time points}

A total of 44,248 genes were detected, and their expression was compared between $s r$ and WT. Among these, 616 DEGs were identified at different time points during the plant response to $P c c$ after inoculation (Fig. 3). The number of DEGs between $s r$ and WT increased from 0 to $12 \mathrm{hpi}$ (36 DEGs at $0 \mathrm{~h}, 60$ DEGs at $6 \mathrm{hpi}, 512$ DEGs at $12 \mathrm{hpi}$ ) and then began to decrease after $12 \mathrm{hpi} \mathrm{(23}$ DEGs at $24 \mathrm{hpi}$ ). At $12 \mathrm{hpi}$, the number of DEGs was the largest, the number of upregulated genes (412) was greater than that of downregulated genes (91), and the expression of defense responses was greater than that at all other time points.

\section{KEGG pathway functional enrichment analysis of the DEGs at $12 \mathrm{hpi}$}

Based on the previous analysis, 12 hpi was the most important defense regulation time point to $P c c$ in Chinese cabbage. KEGG enrichment analysis was performed between $s r$ and WT at $12 \mathrm{hpi}$. A total of 391 DEGs were mapped to 72 KEGG pathways and included those KEGG pathways most significantly identified, including several pathways related to immune response against pathogens (Fig. 4, Supplementary Table S3).

Ten DEGs were enriched in the glucosinolate biosynthesis pathway (Ath00966, 10/19), which was the most highly represented pathway. Twenty-one DEGs were enriched for the term plant hormone signal transduction (Ath04075, 21/271). Twelve DEGs were enriched for the term plant-pathogen interactions (Ath04626,12/164) and included genes that activated the defensive PAMPS. These genes were PTI, including a receptor-like kinase (SERK4), calcium-dependent protein kinase (CDPK), mitogen-activated protein kinase (MPK), and WRKY transcription factor 33 (WRKY33), which regulated resistance to saprophytic bacteria. A total of 51 DEGs were enriched for the term biosynthesis of secondary metabolites (Ath01110, 51/ 995), which contained the phenylpropanoid biosynthesis pathway, and were involved in the synthesis of lignin. Among the upregulated genes identified were phenylalanine ammonia-lyase (PAL), cinnamoyl CoA reductase (CCR), caffeoyl-CoA O-methyltransferase (CCoAOMT), caffeic acid 3-O-methyltransferase 1 (COMT1), and cinnamyl alcohol dehydrogenase (CAD).

To validate the reliability of the resistance-responsive gene expression from RNA-Seq, 16 genes were confirmed based on previous analyses by quantitative realtime PCR using gene-specific primers (Supplementary Table S4).

The expression patterns of the selected resistanceresponsive genes identified by $\mathrm{RT}-\mathrm{qPCR}$ were largely consistent with the RNA-Seq data (Supplementary Fig. 3) and indicated that there was a high degree of agreement in the expression patterns between qPCR and RNA-Seq. $\mathbf{a}$

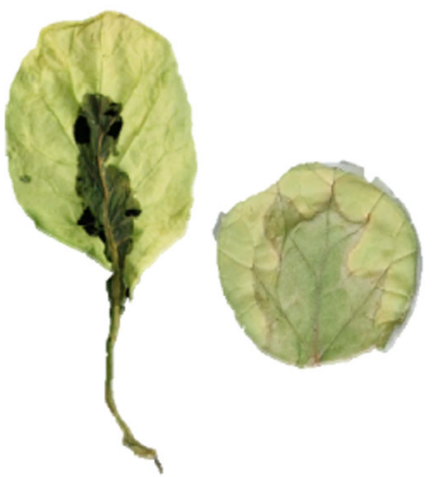

b

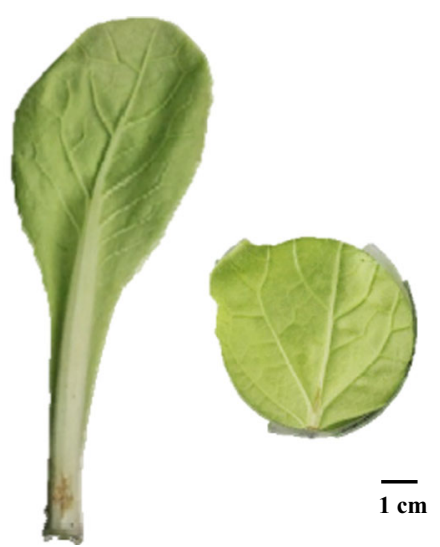

Fig. 2 Disease symptoms in susceptible WT and resistant sr. a The phenotype of WT infected with PCC was 9 in vivo and in vitro. $\mathbf{b}$ The phenotype of $s r$ infected with PCC was 1 in vivo and in vitro 


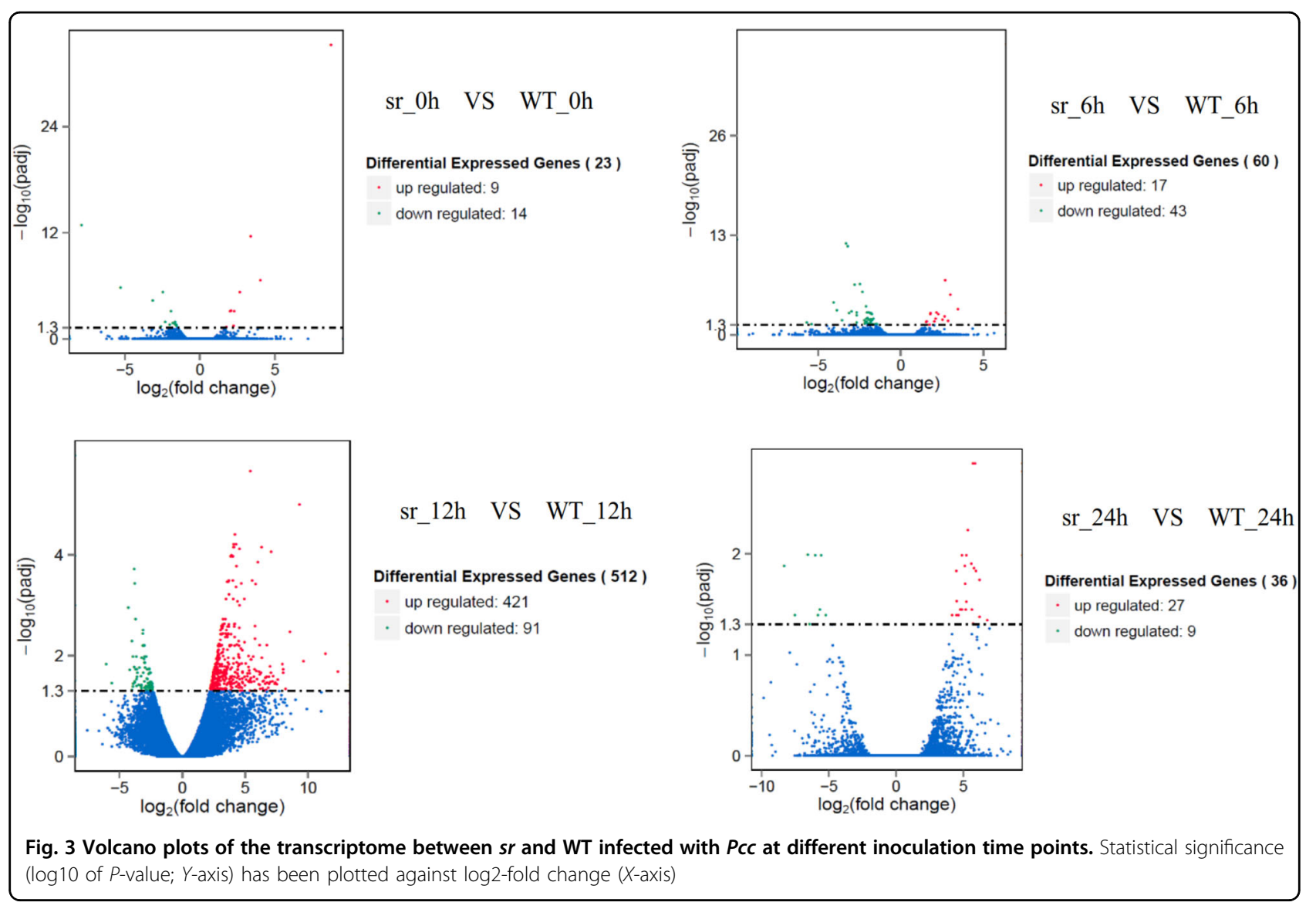

Measurement of glucosinolate in the defense response

Glucosinolate in $s r$ and WT was measured at 0 and 12 hpi (Fig. 5). Eight types of glucosinolate were detected, including three aliphatic glucosinolates: 2-hydroxy-3butenyl (PRO), 3-butenyl (NAP) and 4-pentenyl (GBN); four indolic glucosinolates: 3-indolmethyl (GBC), 1-methoxy-3-indolylmethyl (NEO), 4-hydroxy-3indolylmethyl $(4 \mathrm{OH})$ and 4-methoxy-3-indolylmethyl (4ME); and one benzenic glucosinolate: 2-phenylethyl (NAS). No significant difference in the concentration of NAS between $s r$ and WT was observed between noninoculated plants and those inoculated with Pcc. However, significant differences in the amounts of aliphatic glucosinolate and indolic glucosinolate between sr and WT were observed. The total content of the two compounds increased in $s r$ and WT when inoculated with $P c c$ but was significantly greater in $s r$ compared to WT. PRO was the main component of aliphatic glucosinolate and represented the greatest change in $s r$ at 12 hpi. NAP and GBN expression were very low in Chinese cabbage and was reported to be low in B. napus ${ }^{43}$. However, the content of NAP and GBN significantly increased after 12 hpi with $P c c$, and GBN significantly increased in $s r$ compared to WT. In contrast, there was no difference between $s r$ and WT at 12 hpi because $s r$ contained more NAP than WT at
0 hpi. The absolute increase was larger in WT, which may be due to PRO generated by the hydroxylation of side chains from NAP in the biosynthesis process ${ }^{44}$. There were no significant differences in four types of indolic glucosinolate before inoculation between $s r$ and WT, but they were induced to increase in $s r$ and WT $12 \mathrm{hpi}$ with Pcc. Among these, GBC and NEO were not significantly different between WT and $s r$. The $4 \mathrm{OH}$ and $4 \mathrm{ME}$ forms of indolic glucosinolate increased significantly in $s r$ but not in WT at $12 \mathrm{hpi}$. Therefore, PRO, GBN, $4 \mathrm{OH}$, and $4 \mathrm{ME}$ were determined to be "defense glucosinolate."

\section{Quantitative analysis of lignin after infected Pcc}

The acetyl bromide reaction method was used to detect lignin in the proximal petiole (including the infected wound) and in the leaf (excluding the infected wound) in $s r$ and WT. The analyses of $s r$ and WT were carried out at 0 and 12 hpi (Fig. 6). Because the degree of lignification varies in specific tissues, the lignin content in petioles was higher than in leaf blade. The mean lignin content in the blades and petioles significantly increased in both $s r$ and WT at 12 hpi with Pcc, but the rate of increase in $s r$ blades and petioles was $76 \%$ and $67 \%$, respectively, and greater than that in WT blades and petioles, at $48 \%$ and $47 \%$, respectively. 


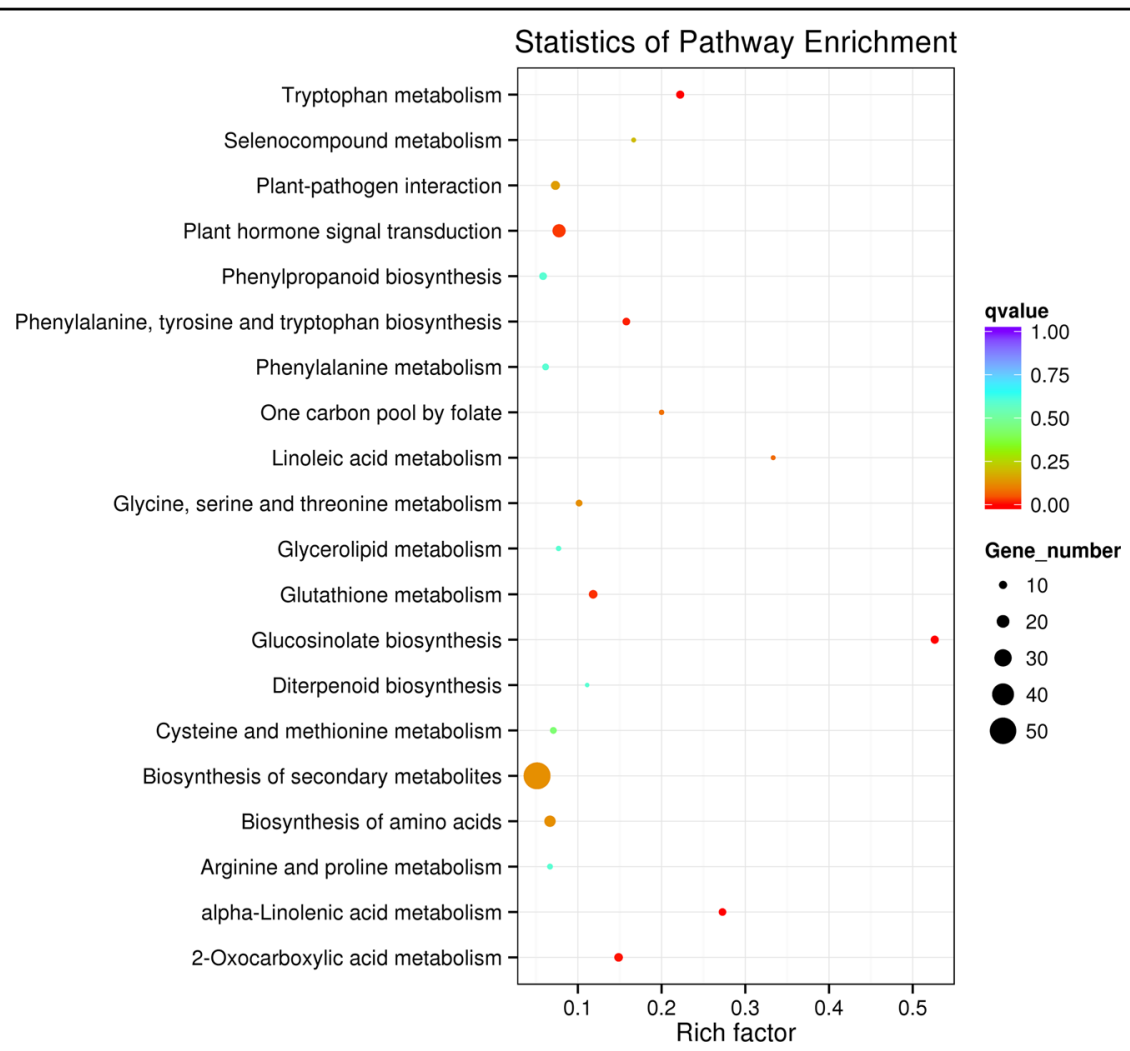

Fig. 4 KEGG functional enrichment analysis for the differentially expressed genes (DEGs) infected with PCC in sr and WT at 12 hpi
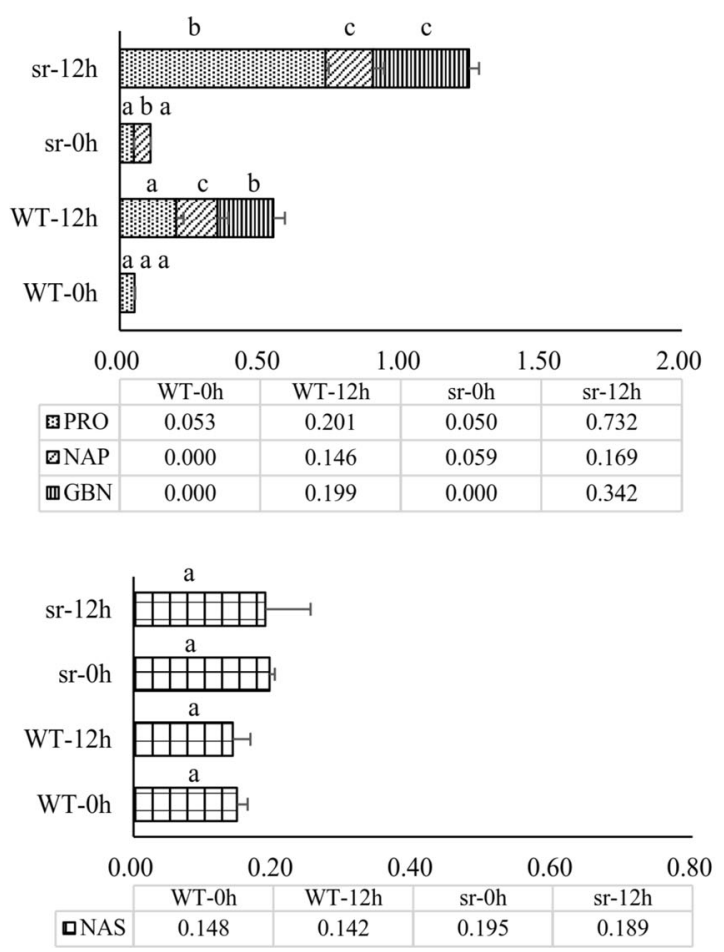

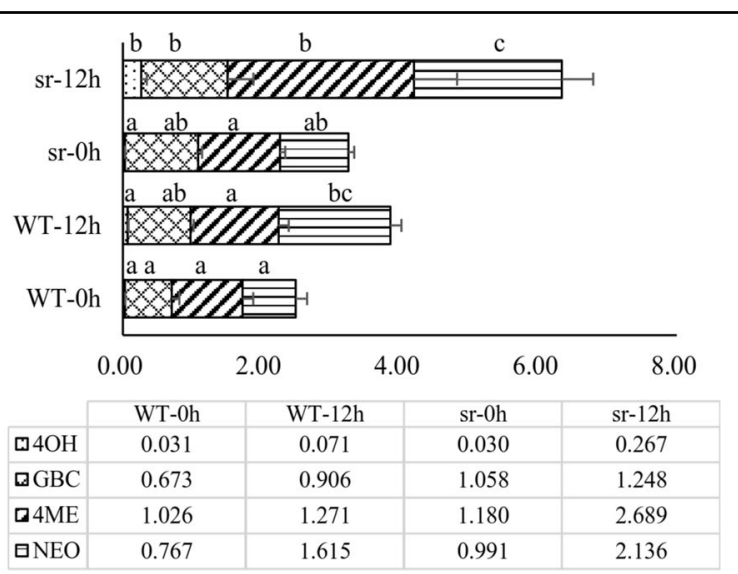

Fig. 5 Glucosinolate levels infected with Pcc in sr and WT at $\mathbf{0 ~ h}$ and $\mathbf{1 2} \mathbf{~ h p i . ~ D a t a ~ r e p r e s e n t ~ t h e ~ m e a n ~ o f ~ a t ~ l e a s t ~ t h r e e ~ r e p l i c a t e ~ s a m p l e s , ~ a n d ~}$ error bars represent the SD. Different letters above the bars denote statistically significant differences $(P<0.05)$ 

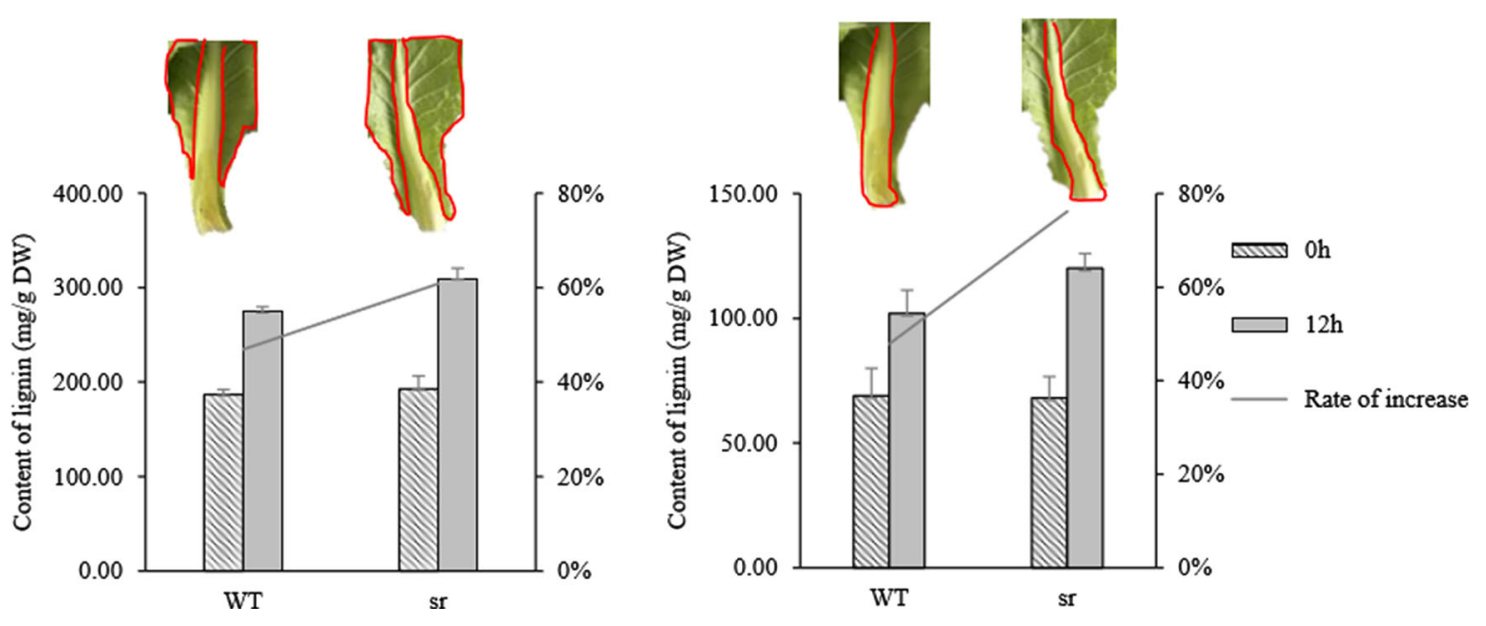

Fig. 6 Lignin content of petiole and leaf infected with Pcc in sr and WT at $0 \mathrm{~h}$ and $12 \mathrm{hpi}$. Lignin was detected in the areas surrounded by red lines. Error bars represent the SD with five biological replicates

\section{Comparison of endogenous auxins, JAs and SA in sr and WT}

Indole-3-acetic acid (IAA) and its derivatives (methyl indole-3-acetate (ME-IAA), 3-indolebutyric acid (IBA), and indole-3-carboxaldehyde (ICA)) were detected in both sr and WT plants (Fig. 7a). ME-IAA level was not affected in $s r$ or WT after inoculation with Pcc. However, 12 hpi with $P c c$, the primary auxin, IAA, decreased in both plant types. Compared to $s r$, IAA level in WT was higher at $0 \mathrm{hpi}$ and decreased to the same level as $s r$ after $12 \mathrm{hpi}$. IBA and ICA levels increased under pathogen stimulation in WT but decreased in $s r$ when the resistant host plant was invaded by pathogen.

Four JAs were present in the host plants (Fig. 7b). MEJA and H2JA were constant during the course of disease development, but JA and JA-ILE increased significantly in $s r$ and WT at 12 hpi with Pcc. The JA level significantly increased in the resistant genotype compared to the susceptible genotype. JA-Ile had similar patterns of JA-Ile to the JA patterns in response to Pcc (Fig. 7b). Pathogens triggered the host plant to increase JA biosynthesis in either susceptible or resistant plants during early infection; however, there was a significantly higher expression level in resistant plants ${ }^{45}$. SA levels showed opposite patterns in $s r$ and WT after 12 hpi with Pcc (Fig. 7c). SA levels significantly increased in $s r$ and significantly reduced in WT, although the SA basal level was higher in WT without the pathogen. The SA level was lower in WT compared to $s r$ at $12 \mathrm{hpi}$ and was similar to the level in $s r$ at $0 \mathrm{hpi}$. In Arabidopsis, the IAA-dependent pathway may have an antagonistic effect on the SA-dependent defense pathway-pathogen interaction ${ }^{46}$. In our study, the SAdependent and IAA-dependent pathways did not show any obvious antagonistic interactions and were opposite to the IBA and ICA patterns.
Effects of exogenous hormone on resistance against Pcc

After the application of exogenous hormones, resistance against $P c c$ significantly changed in $s r$, WT and 'Huaguan' (Fig. 8). IBA application significantly enhanced susceptibility of $s r$ and 'Huaguan' compared to application of IAA. JA retarded disease development in WT and 'Huaguan' but did not completely relieve the disease symptoms. The effect of BTH application inhibited symptom development on leaves regardless of the disease grade of plants. IAA and IBA negatively regulated the immune response against $P c c$, and IBA significantly promoted disease development and enhanced susceptibility. MEJA and BTH positively affected resistance against Pcc.

\section{The putative resistance mechanism to Pcc in Chinese cabbage}

A previous analysis demonstrated that 6 to $12 \mathrm{hpi}$ was the most important defense regulation period against $P c c$ in Chinese cabbage (Fig. 3), and KEGG enrichment analysis in $s r$ at 0 and $12 \mathrm{hpi}$ revealed the putative mechanism of response to Pcc. In $s r, 7747$ DEGs (3579 upregulated genes, 4168 downregulated genes) were mapped to 121 KEGG pathways at $12 \mathrm{hpi}$. Four pathways (glucosinolate biosynthesis, plant-pathogen interaction, plant hormone signal transduction and phenylpropanoid biosynthesis) and related pathways were selected to explain defense mechanisms against Pcc (Supplementary Table S5).

We verified that glucosinolate has an important role in defense against $P c c$, as 15 DEGs were enriched in the glucosinolate biosynthesis pathway (Ath00966, 15/19), which is probably a part of defense against pathogen and insect infection in Brassicaceae plants ${ }^{47}$. Thirty-eight genes were involved in the glucosinolate biosynthesis pathway in Arabidopsis, and 87 genes were described in our study. Some of 


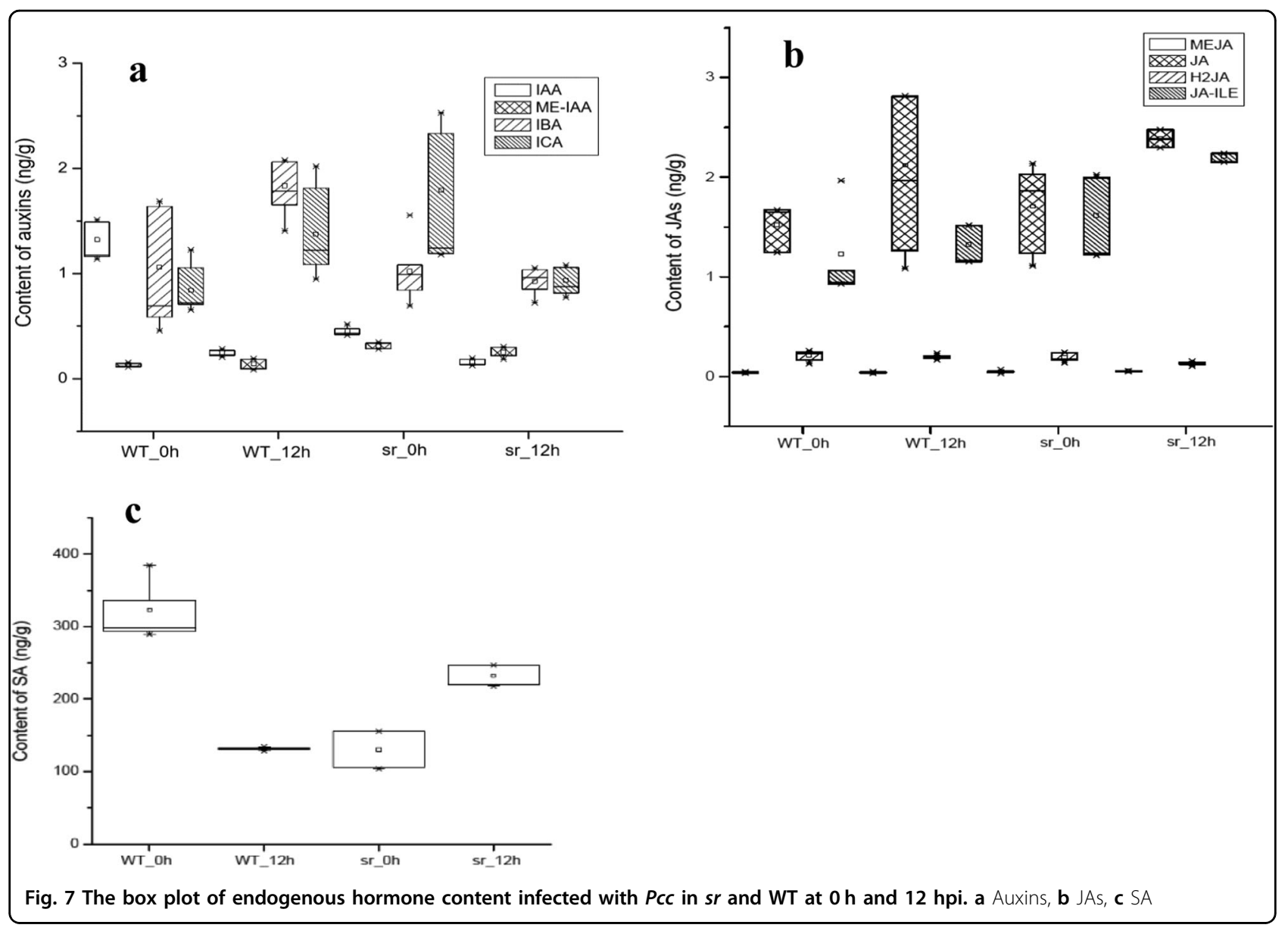

these genes may be homologous to those in Arabidopsis and combined with glucosinolate for defense against $P c c^{29,48-50}$. In our study, 46 of 87 genes were expressed to synthesize "defensive glucosinolate" in aliphatic, indolic and benzenic glucosinolate pathways through the following three phases: side-chain elongation, core structure formation, and secondary modification. The genes in these phases were regulated by transcription factors (Supplementary Table S6, and Fig. 9a). Three types of aliphatic glucosinolates (PRO, NAP and GBN) that were classified by side carbon chain length were detected in our study (Supplementary Table S7, and Fig. 9b). $n=4$ and $n=5$ represented aliphatic glucosinolate with 4 and 5 carbon chains, respectively, in their core structure. The concentrations of PRO, NAP and GBN were stimulated by Pcc, and those of PRO and GBN were significantly higher in $s r$ compared to WT (Fig. 5). GS-OH is responsible for converting NAP to PRO and was upregulated in $s r$ at $12 \mathrm{hpi}$ (Fig. 9c). However, the production of NAP was dependent on $A O P 2$, but three of the AOP2 homologous genes (BrAOP2) were not expressed in our study, and the $A O P 3$ gene was not found in B. rapa. Nevertheless, two of three BrAOP1 genes showed significant changes when plants were challenged with $P c c$, and only one BrAOP1 gene (Bra000847) was upregulated by challenge with Pcc. All genes involved in the indolic and benzenic glucosinolate synthesis pathways were significantly upregulated, except for two MYB transcription factors (BrMYB34-Bra029349, BrMYB51-Bra025666, Fig. $9 \mathrm{a})$. The key genes for core structure formation and secondary modification were upregulated, and gene expression level increases were greater in the resistant mutant $s r$. Limited by the sensitivity of detection technology, only one benzenic glucosinolate (NAS) was formed (Fig. 5). Whether benzenic glucosinolate was produced as a defensive compound is difficult to ascertain. Pfalz et al. ${ }^{51}$ demonstrated that multiple genes control secondary modification to form various indolic glucosinolates. However, $1 \mathrm{OH}-\mathrm{I} 3 \mathrm{M}$ was not detected in our study, and $\mathrm{GBC}, \mathrm{NEO}, 4 \mathrm{OH}$, and $4 \mathrm{ME}$ increased only when the plant was infected (Fig. 5). The CYP81F family of enzymes catalyzed GBC in the first step of modification, and CYP81F2, CYP81F3, and CYP81F1 catalyzed $\mathrm{GBC}$ to $4 \mathrm{OH}$. CYP81F4 was responsible for the conversion of $\mathrm{GBC}$ to $1 \mathrm{OH}-\mathrm{I} 3 \mathrm{M}$. $4 \mathrm{OH}$ and $1 \mathrm{OH}-\mathrm{I} 3 \mathrm{M}$ were converted to $4 \mathrm{ME}$ and NEO through the function of IGMT1 and IGMT2 (Fig. 9b). Therefore, because most of these genes were upregulated, it was verified that $4 \mathrm{OH}$. 


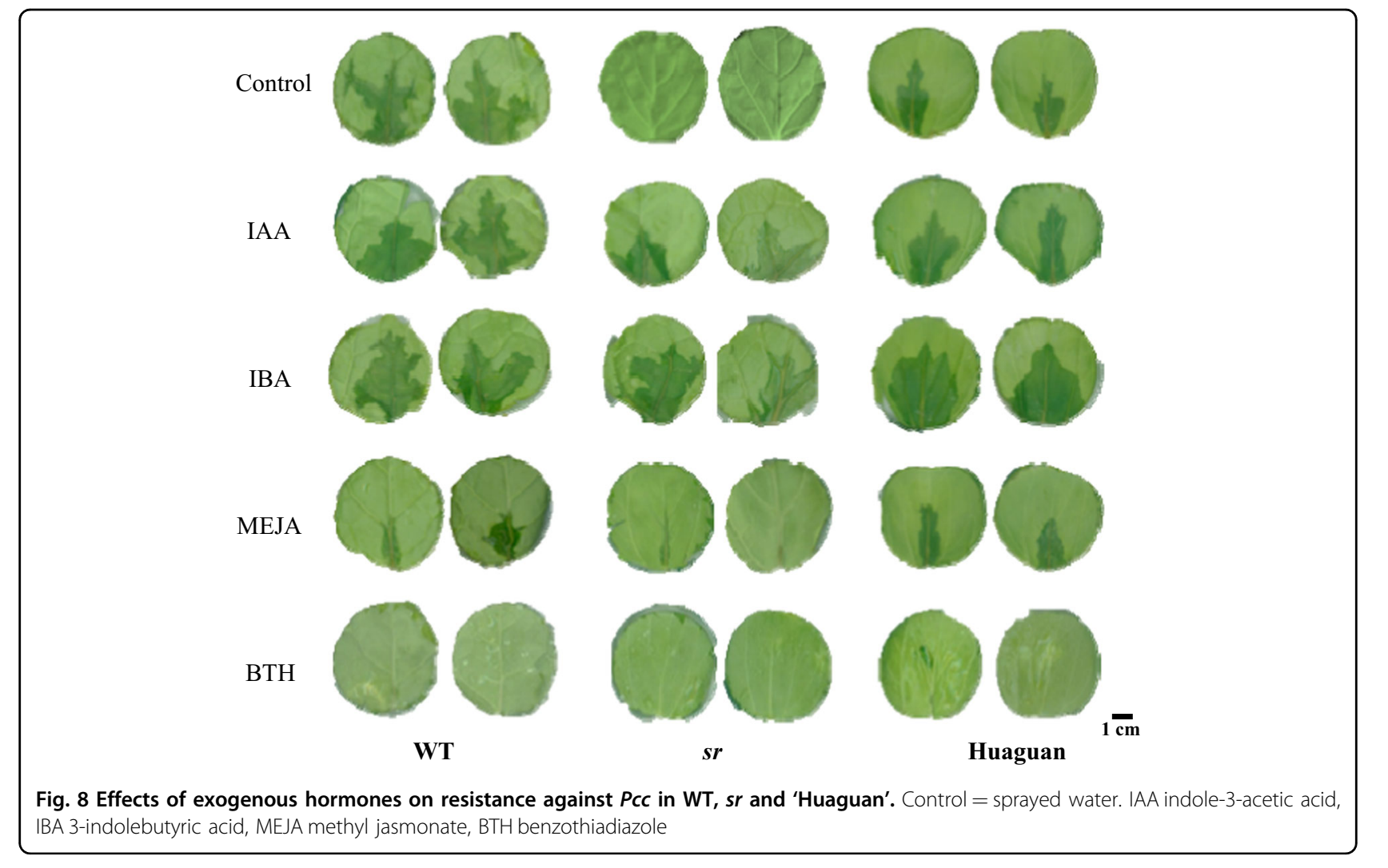

Plants activate the immune response via pathogen recognition and signal transduction at $12 \mathrm{hpi}$ (Ath04626,53/164; Ath04075,142/271). PRRs on the surface of cell membranes recognized M/PAMPs or DAMPs and cause a series of responses ${ }^{11}$. Interestingly, there were no DEGs encoding putative PRRs found at other time points (6 and $24 \mathrm{hpi}$ ). Hence, five putative receptors recognized as M/PAMPs or DAMPs to Pcc triggered the defensive response in our study. Chitin elicitor receptor kinase 1 (CERK1, Brcerk1-Bra031293), chitin receptor (LYM1, Brlym1- Bra016402), and leucine-rich repeat receptor-like protein kinase (PEPR1, Brpepr1- Bra003858) are recognition receptors and had significantly higher expression in $s r$ at 12 hpi than in WT (Figs. 10a and 11). Although the other BAK1-LIKE1/SERK4 (BKK1/SERK4, Brbkk1/serk4-Bra040899) genes were not annotated in the pathway, their function may be part of a receptor complex for different D/PAMPs ${ }^{52}$, whose expression was also higher at $12 \mathrm{hpi}$. The other receptors were WAKs (wallassociated receptor kinases; Brwak2-Bra012273, Brwak4Bra012272) and had been identified as oligogalacturonide (OG) receptors $^{53}$. Eight genes encoding putative polygalacturonase-inhibiting proteins (PGIPs; BrPGIP1Bra009234, Bra009235, Bra009236, Bra009237, Bra009238; BrPGIP2- Bra005917) were upregulated in $s r$ infected with
Pcc. After recognizing M/PAMPs or DAMPs, the downstream defense responses strongly activated the $\mathrm{Ca}^{2+}$-mediated resistance response, eliciting calciumdependent protein kinases (CPKs; Brcpk-Bra008879, Bra023367, Bra031055, Bra018504, Bra001789, Bra000684, Bra037277, Bra034407, Bra009420, Bra001789), calciumbinding proteins (CMLs; Brcml-Bra016936, Bra021379, Bra012889, Bra019503, Bra013470, Bra005797, Bra015728, Bra006595, Bra027848, Bra033745, Bra039511), and respiratory burst oxidase homologs (RBOHs; BrrbohBra037520, Bra013862, Bra027764) to reinforce the cell wall. Concomitantly, mitogen-activated protein kinases (MPK3 and MPK4; Brmpk3-Bra038281 and Brmpk4Bra000955) were activated, interacted with the downstream transcription factors WRKY33 and WRKY25 (Brwrky33-Bra017117, Bra005104, Bra000064; Brwrky25Bra022786, Bra021623) and increased in $s r$ at $12 \mathrm{hpi}$. Ultimately, basic chitinases (PR-3 and PRB1; Brpr3Bra011464 and Brprb1-Bra013123) were upregulated at 12 hpi and were part of the immune response to Pcc (Fig. 10b).

JAs are produced by a series of enzymatic reactions that begin with $\alpha$-linolenic acid as the initial substrate, and the expression of several genes was changed at the transcription level in alpha-linolenic acid metabolism 


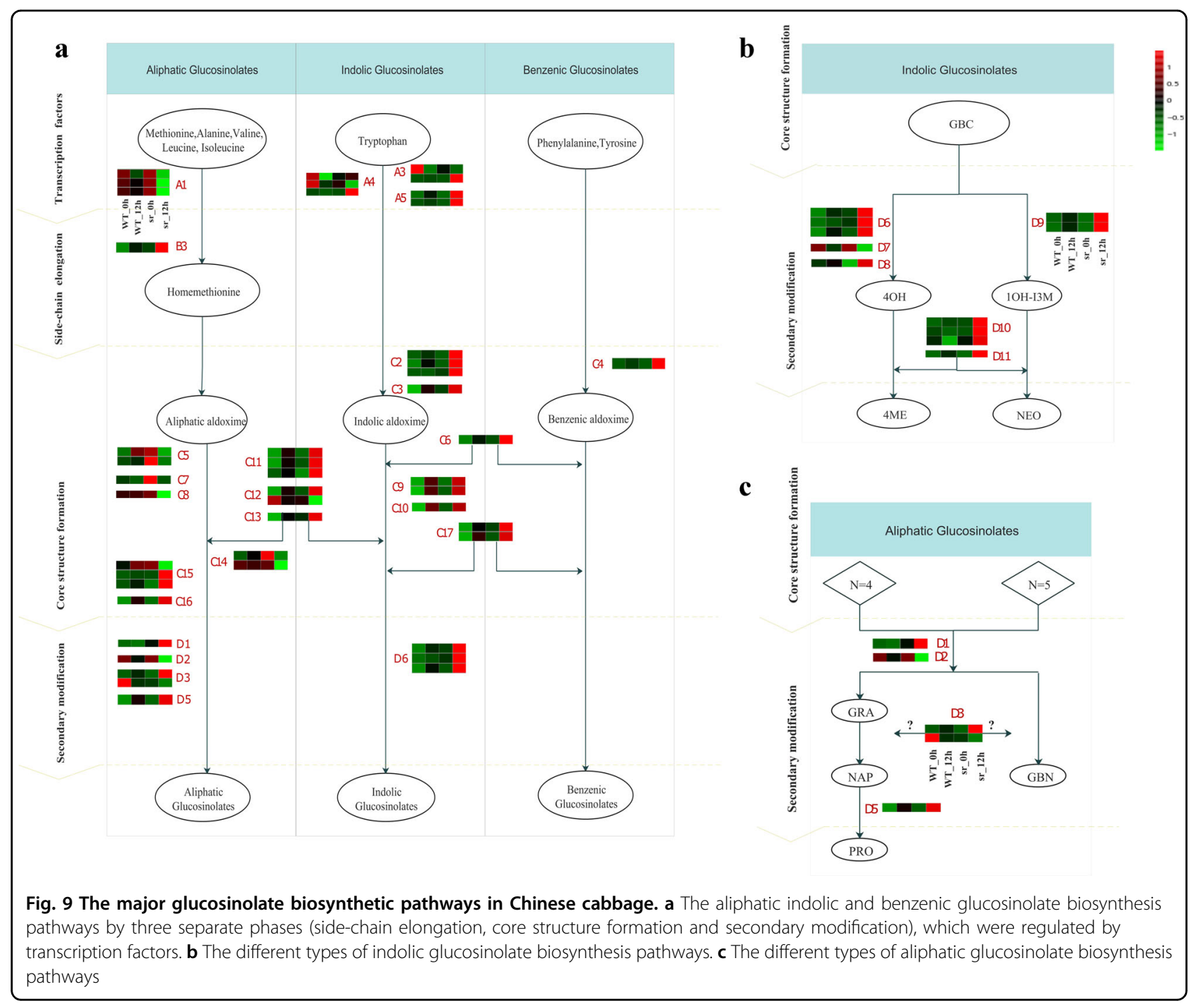

(Ath00592, 25/33) at $12 \mathrm{hpi}$. Among them, the key genes of JA biosynthesis are AOS (Braos-Bra035320), AOC2 (Braco2-Bra028805), AOC3 (Braoc3-Bra017350), LOX2 (Brlox2-Bra030988, Bra016602), and LOX3 (Brlox3Bra030988, Bra016602), which were upregulated in $s r$ (Fig. 10c). At the same time, JA and JA-ILE increased in $s r$ and WT 12 hpi with Pcc. The JA level was markedly increased in resistant plants compared to in susceptible plants (Fig. $8 \mathrm{~b})$. The accumulation of JAs and JA derivatives after inoculation with $P c c$ has been involved in the immune responses of the host plant ${ }^{45}$. The regulation of various enzymes in cysteine and methionine metabolism (Ath00270,51/99), ACS2 (Bracs2-Bra033261), and EFE (Brefe-Bra015380) was attributed largely to the control of ET synthesis. Transcriptional regulation and protein expression in our study were upregulated in the resistant mutant sr. ERF2 (Brerf2- Bra017495) is a shared point between the JA and ET pathways and activated JA/ET downstream regulated genes ${ }^{54}$, which increased in $s r$ but not in WT. However, PDF1.2, HEL, and CHIB are required in the JA/ET signaling pathway to respond to Pcc but were not identified. In our study, SA levels significantly increased in $s r$ but were significantly reduced in WT (Fig. 8c). Unlike JA/ET, there were no SA biosynthesis-related genes found in DEGs. However, WRKY70 (Brwrky70- Bra014692), as a central component in SA signaling, was upregulated to promote the expression of downstream genes in $s r$ but not in WT. IBA and ICA increased in the susceptible WT when inoculated with the pathogen (Fig. 8a). Other evidence showed that exogenous auxin (IAA and IBA) significantly enhanced susceptibility in WT to Pcc (Fig. 9). After inoculation with Pcc, Aux/IAA genes (such as Briaa7-Bra033886, Bra001900; Briaa19-Bra027232, Bra021117) and TIR1 (Brtir1-Bra014378, Bra003518) were inhibited in sr, but seven GH3 family genes (Brgh3.1- Bra039832; Brgh3.2- 


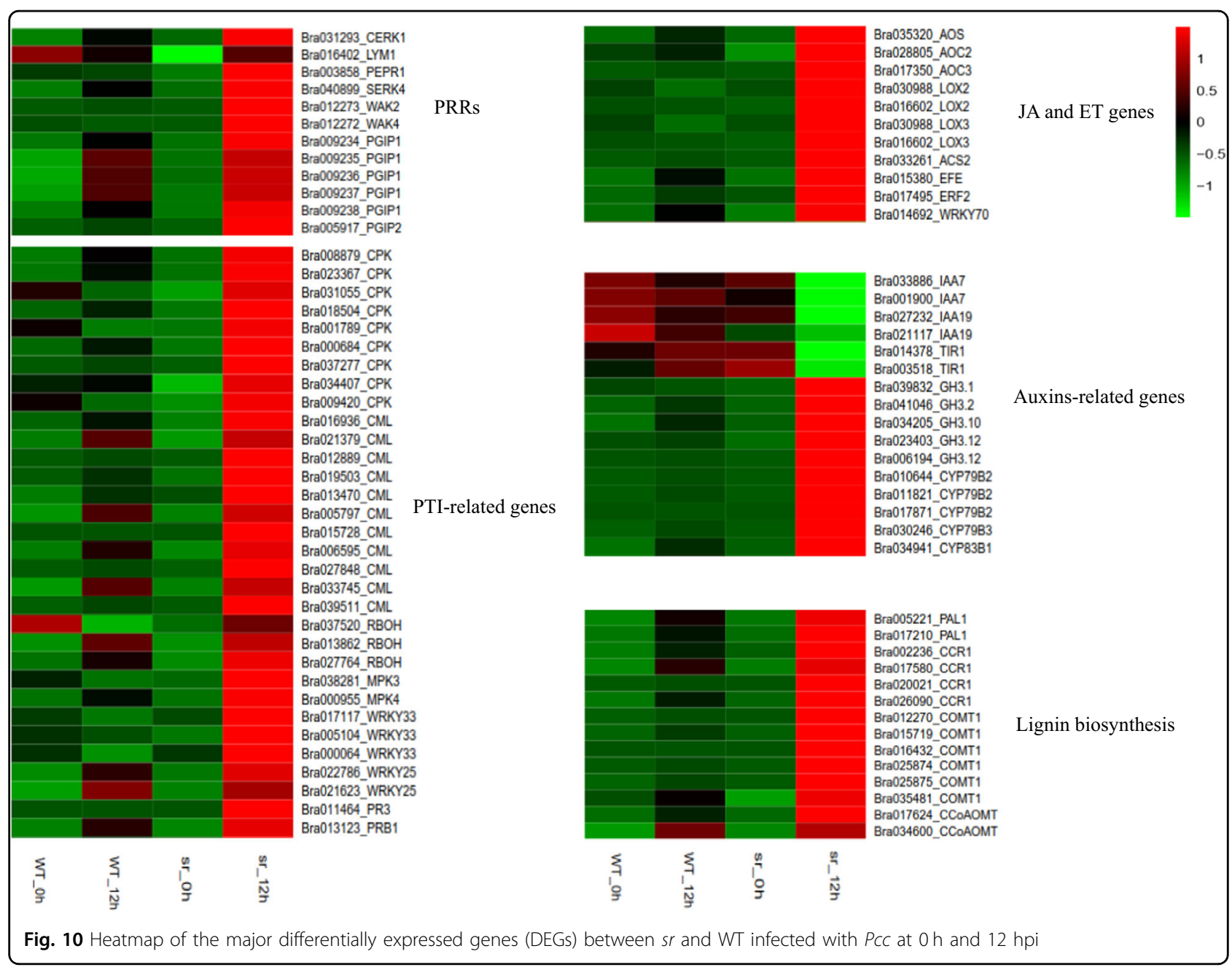

Bra041046; Brgh3.10-Bra034205; Brgh3.12-Bra023403, Bra006194) were upregulated (Fig. 11d). Four GH3 family genes (BrGH3.1, BrGH3.2, BrGH3.10, BrGH3.12) were also upregulated in $s r$ but not in WT at $12 \mathrm{hpi}$. In contrast, primary auxin (IAA) shared a common biosynthetic pathway with indolic glucosinolate and camalexin, making IAOx a regulatory branch point. CYP79B2 (Brcyp79b2Bra010644, Bra011821, Bra017871) and CYP79B3 (Brcyp79b3-Bra030246) were upregulated in $s r$, which promoted the biosynthesis of IAOs in the indole glucosinolate, auxin and camalexin biosynthesis pathways. Indole glucosinolate was synthesized directly from IAOx by CYP83B1 (Brcyp83b1-Bra034941) and was also upregulated. However, there was no significant difference in the expression of Brcyp1a13 and Brcyp71b15, which regulate the synthesis of camalexin. The genes that control the generation of auxin from IAOx are not known.

Lignin synthesis pathway genes were enriched in the term biosynthesis of secondary metabolites (Ath01110, 505/995) at $12 \mathrm{hpi}$. Our study indicated that the expression of genes encoding PAL1 (Brpal1-Bra005221, Bra017210), CCR1
(Brccr1-Bra002236, Bra017580, Bra020021, Bra026090), COMT1 (Brcomt1- Bra012270, Bra015719, Bra016432, Bra025874, Bra025875, Bra035481), and CCoAOMT (Brccoaomt-Bra017624, Bra034600) was upregulated in $s r$ but not in WT (Fig. 10e), and the lignin content of the cell wall of $s r$ was increased after 12 hpi with Pcc (Fig. 6). The accumulation of lignin could provide a positive defense effect against $P c c$.

\section{Discussion}

Fitness of disease severity scoring method and resistance period in the immune system

In this study, the disease severity of soft rot was evaluated in vitro and in vivo for lesion size in Chinese cabbage (Fig. 1 and Supplementary Fig. 1). Because of low humidity and the speed of disease development, plants could be scored in vivo at $48 \mathrm{hpi}^{34}$ and in vitro at $24 \mathrm{hpi}^{28}$, which made the disease severity accurate but also met the requirements of harvesting samples for RNA-Seq analysis. The transcript profiles were investigated with $s r$ and WT at $0 \mathrm{~h}, 6,12$, and $24 \mathrm{hpi}$ in 


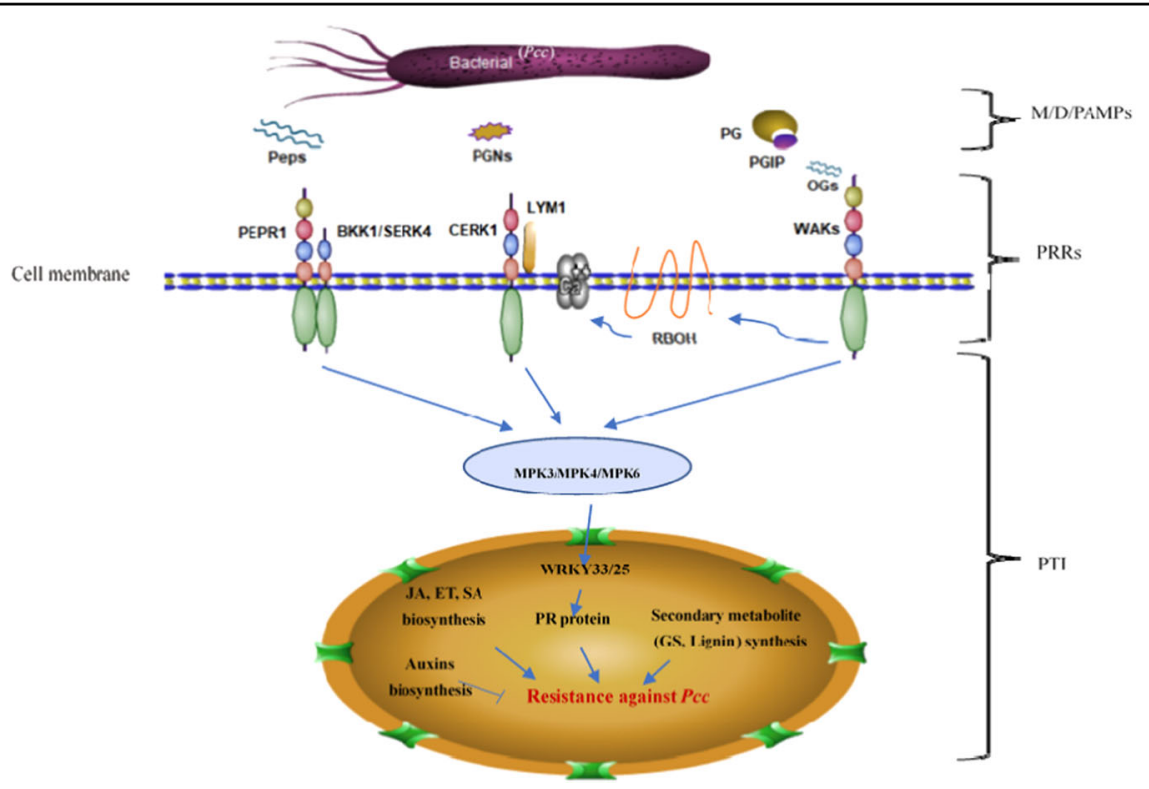

Fig. 11 Simple schematic diagram of the interactions between Pcc and Chinese cabbage in our study. The major bacterial M/D/PAMPs (Peps, PGNs, and OGs) were recognized by different PRRs (BrBKK1/SERK4-PEPR1, BrLYM1-BrCERK1, and BrWAKs) to activate immune responses. MAPK activation is an important component of PTI signaling. Two major WRKY transcription factors (WRKY25 and WRKY33) are also targets of MAPK phosphorylation, which regulates PR protein activity. JA, ET, and SA were induced to accumulate transduction signals, and auxins were affected as some negative signals. Glucosinolate and lignin, as secondary metabolites, were synthesized and had functional roles in defense against PCc

response to Pcc using RNA-Seq. The petiole, not the leaf blade, was inoculated and used for in vivo samples. Leaf blades had not been in contact with the pathogen during $6 \mathrm{hpi}$, and the mutant $s r$ showed the strongest resistance at $12 \mathrm{hpi}$ and remained resistant at $24 \mathrm{hpi}$. In contrast, WT did not incite protection against $P c c$ at $12 \mathrm{hpi}$, and macerated lesions appeared at $24 \mathrm{hpi}$. Therefore, 6-12 hpi was the initial defense regulation period to $P c c$ in our study.

\section{The putative immune mechanism of the Chinese cabbage-} Pcc interaction

Mutated genes for soft rot resistance traits were identified from the $F_{2}$ population (two parents: resistant mutant $s r$ and WT) by the MutMap method ${ }^{55}$ (data are unpublished). Considering that the F1 plants showed susceptibility to $P c c$ and that disease severity segregated into susceptibility and resistance at a segregation ratio of 3:1 in the $\mathrm{F}_{2}$ population, the resistant mutant trait may be controlled by a single recessive locus. A subset of 5 genes having nonsynonymous SNPs was chosen in resistant mutant $s r$ (Supplementary Table S8).

There are three separate modes of action in plant innate immunity responses: ETI, PTI and systemic acquired resistance (SAR), and they are obviously different and closely correlated to interact with pathogens ${ }^{11}$. BTH, as a substitute for SA, maintained a longer chemical effect than did SA and was repeatedly shown to be effective against pathogens by activating the SAR pathway ${ }^{56}$. The effects of BTH application on enhanced resistance were significant against $P c c$ in susceptible Chinese cabbage and tolerant pak choi (Fig. 8). These results demonstrated that BTH treatment could trigger SAR in the host plant to enhance immunity.

In our study, ETI was not the primary defensive strategy of the host plant against Pcc. However, PTI appears to have a central role in plant defense against $P c c$, which is consistent with the review of Davidsson et al. ${ }^{9}$. We found three putative R-structure genes (Bra013144, Bra027047, Bra037141) from DEGs at $12 \mathrm{hpi}$, but these genes did not occur at other time points, and their expression also increased in the susceptible WT from 0 to $24 \mathrm{hpi} \mathrm{(Fig.} \mathrm{5).}$ ETI triggered immune responses with PCD to cause HR and enabled necrotrophic pathogens to acquire more nutrients from dead plant tissues and promote advancement of the infection. However, PCD has the opposite effect on resistance in biotrophic pathogens because it can restrict the growth and colonization of pathogens ${ }^{9,14}$. In our study, the expression of key genes of $\mathrm{PTI}^{57,58}$, mitogen-activated protein kinase (MPK), calcium-binding protein $(\mathrm{CML})$, calcium-dependent protein kinase (CPK), respiratory burst oxidase homolog $(\mathrm{RBOH})$, and WRKY33, increased at $12 \mathrm{hpi}$ in the resistant mutant $s r$ but not in the susceptible WT.

PTI was triggered by three different PPRs: BrLYM1BrCERK1 may comprise PGN recognition, BrBKK1/ 
SERK4-PEPR1 was a receptor complex recognized by BrPeps, and BrWAK2, and BrWAK4 were involved in an immune response against $P c c$ by recognizing DAMPs such as OGs.

Well-known PAMPs are bacterial flagellin (flg22) and elongation factor $\mathrm{Tu}(\mathrm{EF}-\mathrm{Tu})$, which are recognized by plant PRRs, such as flagellin-sensitive 2 (FLS2) and EF-Tu receptor (EFR), and trigger plant defenses to induce PTI against different pathogens ${ }^{59,60}$. Interestingly, the expression of PRRs, such as FLS2 and EFR, did not change over the course of the experiments. Chitin is the main wall compound in fungal cell walls that can be hydrolyzed into chitin fragments by plant chitinases as a defensive mechanism. Chitin elicitor receptor kinase 1 (CERK1) recognizes chitin from the fungal cell wall as a PAMP leading to the expression of $\mathrm{PTI}^{61}$. Peptidoglycans (PGNs) are gram-positive and gram-negative bacterial cell walls whose structures are similar to chitin found in fungi. PGNs are recognized by AtLYM1 and AtLYM3 combined with AtCERK1 in Arabidopsis to trigger PTI ${ }^{62}$. In Chinese cabbage, $P c c$ may release PGNs that were recognized by BrLYM1-BrCERK1 and activated genes to protect the host plant from being infected (Fig. 11).

Endogenous small peptides (Pep1-8) act as M/DAMPs and are recognized by PEPR1 and its homolog PEPR2 to activate PTI to pathogens ROS, and ET is also involved in PEPR signaling ${ }^{63,64}$. BRI1-associated receptor kinase 1 (BAK1/SERK3) and its closest paralogue BAK1-Like1/ SERK4 (BKK1/SERK4) are ligands within other PRRs and form complexes contributing to PTI signaling ${ }^{52}$. Similar to FLS2 and EFR, BAK1/SERK3, the closest paralogue to BKK1/SERK4, is also required to elicit PTI to associate with the PEPR-mediated response signaling system in response to AtPeps ${ }^{64,65}$. Therefore, BrBKK1/SERK4PEPR1 function in a direct role to elicit PTI as part of a receptor complex for some Peps or MAMPs in Chinese cabbage (Fig. 11).

In our study, $B r W A K 2$ and $B r W A K 4$ were identified at $12 \mathrm{hpi}$ and participated in defense against Pcc. WAKs can distinguish and respond to OGs inducing a defense respons $^{53}$ and are degraded products from pectinderived homogalacturonan released from plant cell walls by PCWDs (such as PGs) and function as DAMPs ${ }^{66}$. WAKs bind to two types of pectin: native pectin regulates cell expansion, and one OG activates the response pathway by the pathogen. The binding of WAKs depends on the affinity for the esterified polymers ${ }^{67}$. One assumption was that different WAKs can distinguish types of pectin or OGs formed by different pathogens, and these two types tend to be recognized by different WAKs. Furthermore, eight genes encoding putative polygalacturonaseinhibiting proteins (PGIPs) were upregulated in $s r$ but not in WT (Fig. 10a). One BrPGIPs gene (Bra005918) was considered a candidate gene harboring one nonsynonymous SNP (leucine to glutamine in an exon) in resistant mutant $s r$. PGIPs are PG inhibitor proteins of cell wall-degrading enzymes located in plant cell walls ${ }^{68}$. They combine with PG to inhibit the degradation and maintain the integrity of the plant cell wall. The role of PGIPs is defense against fungal pathogens ${ }^{69}$. However, it was also indicated that it may have a potentially important defense role in Chinese cabbage against $P c c^{26}$.

Regardless of which PRRs recognized M/PAMPs or DAMPs to trigger PTI, downstream defense responses (CPK, CML, RBOH MPK3, and MPK4) were strongly activated during infection at $12 \mathrm{hpi}$ in $s r$ (Fig. 10a). In Arabidopsis, PEPR1 and PEPR2 recognized AtPeps to produce $\mathrm{ROS}^{63}$ and OGs and induced a very strong AtRBOHD-dependent apoplastic ROS burst ${ }^{70}$. These related genes were upregulated in $s r$, but not in WT, and suggested that PTI had a major role in resistance against $P c c$ in the mutant. In our study, three copies of WRKY33 were upregulated in $s r$ but not in WT. The WRKY33 transcription factor is a downstream gene for plant resistance to necrotrophic pathogens ${ }^{24}$. Knockout wrky33 mutant plants are highly susceptible to necrotrophic pathogens, but overexpression of WRKY33 increases resistance to Botrytis and Alternaria brassicicola in Arabodopsis $^{23,24}$. WRKY33 is also a specific regulator of the autophagy gene $A T G 18 a$, which enables the formation of the degradation autophagosome of cytoplasmic components $^{57,71}$. However, ATG18a, which impacts immune responses significantly against $P c c$ through PTI immunity, may not be related to autophagy.

\section{Glucosinolate and auxin shared the same branch point but had the opposite effect on the immune response}

Glucosinolates (GSs) are the products of Brassicaceae species, which are involved in plant defense against insects and pathogens and whose regulatory networks are affected by the plant hormones JA, SA, and ET and by protein kinase and oxidation reduction ${ }^{72,73}$. Indolic glucosinolate is involved in plant growth and defensive responses to pathogens ${ }^{72,74}$. Regardless of the class of glucosinolate, the formation of glucosinolate can be included in the following three separated phases: sidechain elongation, core structure formation, and secondary modification, and the genes in these phases are regulated by transcription factors (Fig. 9a). However, no methylthioalkylmalate synthase family $(M A M)$ genes related to defense after inoculating $P c c$ at the seedling stage were expressed in our study. This family of genes controls the side-chain length of aliphatic glucosinolate and originates from methionine ${ }^{75}$. Only one gene, BCAT-3 (Brcat-3Bra029966), was expressed during the side-chain elongation phase and upregulated significantly in $s r$ at $12 \mathrm{hpi}$, but not in WT. The formation core structure is catalyzed by the CYP79 and CYP83 families that belong to 
cytochrome P450 enzymes. Our results showed that most CYP79 family genes were upregulated in $s r$ induced by $P c c$ (Fig. 10a), which is consistent with research on Arabidopsis $^{74,76}$. The gene CYP79F1, which converted the substrate of phenylalanine and methionine to aldoxime ${ }^{77}$, was not expressed, whereas $C Y P 79 B 2$ and $C Y P 79 B 3$ converted tryptophan to indole-acetaldoxime (IAOx), and CYP79A2 participated in the formation of benzenic glucosinolate, which increased in $s r$ at $12 \mathrm{hpi}$. In our study, the expression of CYP83B1 was upregulated in WT and downregulated in $s r$. CYP83B1 preferentially uses indole3 -acetaldoxime and aromatic aldoximes as substrates, whereas CYP83A1 acts on aliphatic aldoximes ${ }^{78,79}$. In the side-chain elongation phase, some genes acted on two types of glucosinolate. These genes were also upregulated in $s r$ (except one, Brsur1- Bra036703). In our study, all genes were involved in core structure formation and significantly upregulated in the indolic and benzenic glucosinolate synthesis pathways (Fig. 9a), which implied that indolic and benzenic glucosinolates accumulated and had a functional role in defense against $P c c$ in Chinese cabbage.

In our study, GS-OH was upregulated in $s r$ at $12 \mathrm{hpi}$, was responsible for the conversion of NAP to PRO, and explained the accumulated PRO (Fig. 9c). The AOP family has three copies (AOP1, $A O P 2$, and $A O P 3)$, and $A O P 2$ and $A O P 3$ were identified as potential genes in the stage of aliphatic glucosinolate modification ${ }^{80}$. However, three AOP2 homologous genes (BrAOP2) were not expressed in our study, and there was no $A O P 3$ gene in $B$. rapa. Nevertheless, two of the three BrAOP1 genes had significant changes, but only one BrAOP1 gene (Bra000847) had increased expression stimulated by $P c c$. The production of NAP and GBN was dependent on $A O P 2$. AOP1 was considered to be ancestral by tandem repeat production to have $A O P 2$ and $A O P 3$, the biological function in the synthesis of NAP and GBN was not clear ${ }^{81}$. CYP81F family genes were responsible for the conversion of indolic glucosinolate (Fig. 9b). Most of these genes that were upregulated verified that indolic glucosinolate increased significantly in $s r$ at 12 hpi but not in WT. No conclusions can be made as to whether benzenic glucosinolate production participated in this pathogen defense. From this evidence, we explicitly suggest that glucosinolate, especially indolic glucosinolate as a secondary metabolite in $B$. rapa, has a functional role in defense against $P c c$.

Demonstrating that resistance to $P c c$ is due to indolic glucosinolate is difficult because indolic glucosinolates share a common biosynthetic pathway with camalexin and IAA. IAOx is a regulatory branch point that can be degraded into indole acetonitrile (IAN) by CYP1A13, which in turn can be hydrolyzed by nitrilases into IAA and oxidatively decarboxylated into camalexin ${ }^{82}$. Camalexin is a phytoalexin generated by plants under biological or abiotic stress and regulated by cytochrome $\mathrm{P} 450$ enzymes CYP79B2, CYP79B3, CYP1A13, and CYP71B15 ${ }^{83}$. $C Y P 79 B 2$ and $C Y P 79 B 3$ were upregulated in $s r$, which promoted the biosynthesis of IAOs in the indole glucosinolate pathway (Fig. 9). There was no significant difference in the expression of Brcypla13 and Brcyp71b15, which may suggest that camalexin may not be the reason for induction of defense against Pcc. IAA not only negatively inhibits the response to pathogens but also shares biosynthetic pathways with defense compounds and is elevated after pathogen infection ${ }^{84}$. Whether the homeostasis of IAOx, which IAA and indole glucosinolate shared, was broken, more IAOx flowed to the indole glucosinolate biosynthesis pathway to produce more indole glucosinolate for defense against the pathogen.

After inoculating plants with $P c c$, Aux/IAA and TIR1 were inhibited, but some GH3 family genes were upregulated, and the expression pattern was similar to the molecular mechanism of auxin-dependent signaling for defense responses to pathogenesis ${ }^{46}$ (Fig. 10d). In contrast to other IAA genes, not all members of the GH3 gene family inactivate IAA, whereas synthetases modify the action of IAA, SA, or JA by conjugating them to amino acids ${ }^{85}$. Endogenous auxins (IAA, IBA, and ICA) and applied exogenous auxins (IAA and IBA) enhanced the susceptibility of plants to Pcc (Figs. 7a and 8). Interestingly, IBA and ICA patterns are opposite to the SA pattern (Fig. 7c). One question proposed is whether the auxin-dependent pathway exerts an antagonistic effect on the SA-dependent defense pathway in plant-pathogen interactions. Four genes (GH3.1, GH3.2, GH3.10, and GH3.12) were upregulated in $s r$ compared with WT at 12 hpi. In Arabidopsis, GH3-12 acted directly on SA or on a competitive inhibitor of $\mathrm{SA}^{86}$. However, GH3.2 is suppressed by auxin signaling and does not require activation of the SA or JA signaling pathway in rice ${ }^{87}$. The mechanism of GH3.1 and GH3.10 does not clearly affect the response to any hormone signal $^{88}$. Maybe the difference between rice and Arabidopsis results in different mechanisms of inhibition of auxin-dependent defense or the different members of the GH3 family influence a different response pathway. Our results suggest that disease resistance conferred by the suppression of auxin signaling is involved in the SAdependent pathway to activate the defense against $P c c$, but more research is necessary to confirm this hypothesis.

\section{The accumulation of SA, JA, and ET as transduction signals in the defense response}

SA, JA, and ET signaling pathways are independent but also have complex cross-talk interactions among them and are utilized accurately by different mechanisms in 
different plant-pathogen interactions to activate immune responses in plants ${ }^{15,16}$. JAs, including jasmonic acid and methyl jasmonate (MeJA), are lipid-derived hormones that regulate plant development, respond to biological and abiotic stresses and have significant roles in disease resistance against necrotrophic pathogens ${ }^{89}$. Pathogens trigger the host plant to increase JA biosynthesis, and there is a significantly higher level of JA expressed in resistant plants ${ }^{45,90}$. JAs are synthesized with a series of enzymatic reactions that begin with $\alpha$-linolenic acid. LOX, AOS, and AOC are key enzymes involved in the synthesis of JAs, whose expression increased in $s r$ but not in WT (Fig. 10c). The accumulation of JAs after inoculation with Pcc demonstrated their involvement in the immune responses of the host plant (Fig. 7b).

JA/ET signaling pathways interact positively with defense responses against necrotrophic pathogens ${ }^{14,17}$. Although the process of ET biosynthesis involves various regulated enzymes, $A C S$ is largely attributed to the control of ET synthesis via transcriptional regulation and protein expression. In our study, $A C S$ was upregulated in the resistant mutant $s r$, but not WT, and is similar to other studies $^{91}$. ERF is a common point of the JA and ET pathways and activates JA/ET downstream regulated genes $^{54}$, which were increased in $s r$ but not in WT. The PDF1.2, HEL, and CHIB genes are required in the JA/ET signal pathway to respond to $P c c^{18}$ but were not identified in WT and $s r$. Because induction of defense gene expression appears to be achieved by a very complicated combination of signals not only from JA/ET but also from some negative pathway effectors such as IAA, it is not possible to discern which hormone signal systemcontrolled defense response is controlled by these genes. In addition, we suspect that the time points chosen in our experiment were earlier than the hormone signal transduction and that the accumulation of JA and ET were synergistically associated with immunity to $P c c$.

Resistance against $P c c$ can be enhanced by the induction of JA/ET-mediated genes, as demonstrated in our study. Interestingly, SA-mediation was also revealed to be an efficient defense against $P c c^{19,20}$. SA-dependent responses are commonly required for defense against biotrophs ${ }^{17}$. SA increased in the plants following initial infection by pathogens and established SAR with several pathogenesisrelated (PR) genes expressed ${ }^{92}$. In our study, SA levels were significantly increased in $s r$ and concomitantly significantly reduced in WT after Pcc inoculation (Fig. 7c). Furthermore, applications of BTH enhanced resistance significantly against Pcc (Fig. 8). However, cross talk between SA and JA/ET signaling is repressed in the resistant response. WRKY70 is a central component in SA signaling, followed by increased SA and decreased JA signaling, which result in enhanced resistance ${ }^{20}$. In this study, WRKY70 was upregulated in $s r$ to promote downstream genes expressed, but not in WT.

There is apparent controversy regarding whether SA is involved in the response to the Pcc-depressed JAdependent pathway. This could be explained by the different efficacies induced by SA- and JA/ET-dependent pathways. The network of the signaling pathway is extremely complex, and as we expected, a gene could have several roles to defend against pathogens. Furthermore, consistently overlapping the various defensive pathways could be triggered by several genes in different pathways. It is nearly impossible to analyze one gene or signal pathway independently. We suggest that the mechanisms of hormone signals are a joint defense against $P c c$ and include an induced resistance response that requires JA/ ET-dependent signaling pathways. We further hypothesize that SA-dependent pathways participate in resistance to $P c c$ and that auxin-dependent pathways interact with JA/ET and SA pathways to inhibit defensive responses.

\section{Lignin protects against further infection in the immune response}

Lignin is the natural product for the structural integrity of the cell wall, which has a role in mechanical support and water transportation during the development of plants. In plant defense against damage and disease, lignin is formed to prevent nutrient and water loss and the spread of pathogens from the initial point of attack $^{93}$. Lignin is closely associated with the resistance of plants to pathogens, and increased lignin in plants can enhance this resistance $^{94}$ (Fig. 6). The phenylalanine ammonia-lyase (PAL) gene was upregulated in $s r$ but not in WT. PAL is the first enzyme in the phenylpropanoid pathway and is located at the beginning of primary metabolism that leads to secondary metabolism in lignin synthesis. The other genes, including cinnamoyl $\mathrm{CoA}$ reductase (CCR), caffeoyl-CoA O-methyltransferase (CCoAOMT), and cinnamyl alcohol dehydrogenase (CAD), were more highly expressed in $s r$ than in WT (Fig. 10e). Our findings are consistent with Zhang et al. ${ }^{34}$ and demonstrated that lignin protected the host plant from further infection by Pcc.

\footnotetext{
Acknowledgements

We are grateful to Prof. Robert N. Trigiano for critical comments and editing this manuscript. This work was supported by the National Key R\&D of China [Grant No. 2017YFD0101802, 2016YFD0100204-17], the National Natural Science Foundation of China [Grant No. 31672151 and 31772324], the Hundred-Talents Program of Hebei Universities [Grant No. SLRC2017040], the Basic Research Program of Hebei [Grant No. 18966925D], the Natural Science Foundation of Hebei [C2016204170], the Science and Technology Support Program of Hebei [16226304D-2], the Science and Technology Research Project of Hebei Colleges and Universities [Grant No. QN2017084 and ZD2017236], the International Cooperation Project in the Science and Technology Support Program of Hebei [Grant No. 17396315D], and the Graduate Student Innovation Fund Project in Hebei Province [CXZZBS2017067].
} 


\section{Conflict of interest}

The authors declare that they have no conflict of interest.

Supplementary information accompanies this paper at (https://doi.org/ 10.1038/s41438-019-0149-z).

Received: 14 September 2018 Revised: 20 March 2019 Accepted: 31 March 2019

Published online: 01 June 2019

\section{References}

1. Gardan, L., Gouy, C., Christen, R. \& Samson, R. Elevation of three subspecies of Pectobacterium carotovorum to species level: Pectobacterium atrosepticum sp. nov., Pectobacterium betavasculorum sp. nov. and Pectobacterium wasabiae sp. nov. Int. J. Syst. Evol. Microbiol. 53, 381-391 (2003).

2. KIKUMOTO, T. Ecology and biocontrol of soft rot of Chinese cabbage. Jpn. J. Phytopathol. 66, 60-62 (2000).

3. Perombelon, M. C. \& Kelman, A. Ecology of the soft rot erwinias. Annu. Rev. Phytopathol. 18, 361-387 (1980).

4. Abbott, D. W. \& Boraston, A. B. Structural biology of pectin degradation by. Enterobact. Microbiol. Mol. Biol. Rev. 72, 301-316 (2008).

5. Charkowski, A. et al. The role of secretion systems and small molecules in softrot Enterobacteriaceae pathogenicity. Annu. Rev. Phytopathol. 50, 425-449 (2012).

6. Lee, D. et al. Characterization of genes required for the pathogenicity of Pectobacterium carotovorum subsp. carotovorum Pcc21 in Chinese cabbage. Microbiol 159, 1487-1496 (2013).

7. Hogan, C. S., Mole, B. M., Grant, S. R., Willis, D. K. \& Charkowski, A. O. The type III secreted effector DspE is required early in Solanum tuberosum leaf infection by Pectobacterium carotovorum to cause cell death, and requires Wx (3-6) D/E motifs. PLOS ONE 8, e65534 (2013).

8. Kim, H. S., Thammarat, P., Lommel, S. A., Hogan, C. S. \& Charkowski, A. O. Pectobacterium carotovorum elicits plant cell death with DspE/F but the P. carotovorum DspE does not suppress callose or induce expression of plant genes early in plant-microbe interactions. Mol. Plant Microbe Inter. 24, 773-786 (2011)

9. Davidsson, P. R., Kariola, T., Niemi, O. \& Palva, E. T. Pathogenicity of and plant immunity to soft rot pectobacteria. Front. Plant Sci. 4, 191 (2013).

10. Parker, J. E. Plant recognition of microbial patterns. Trends Plant. Sci. 8, 245-247 (2003).

11. Jones, J. D. \& Dangl, J. L. The plant immune system. Nature 444, 323-329 (2006).

12. Greenberg, J. T. \& Yao, N. The role and regulation of programmed cell death in plant-pathogen interactions. Cell Microbiol. 6, 201-211 (2004).

13. Macho, A. P. \& Zipfel, C. Targeting of plant pattern recognition receptortriggered immunity by bacterial type-III secretion system effectors. Curr. Opin. Microbiol. 23, 14-22 (2015)

14. Mengiste, T. Plant immunity to necrotrophs. Annu. Rev. Phytopathol. 50, 267-294 (2012)

15. Kunkel, B. N. \& Brooks, D. M. Cross talk between signaling pathways in pathogen defense. Curr. Opin. Plant. Biol. 5, 325-331 (2002).

16. Yang, Y. X., Ahammed, G. J., Wu, C., Fan, S. Y. \& Zhou, Y. H. Crosstalk among jasmonate, salicylate and ethylene signaling pathways in plant disease and immune responses. Curr. Protein Pept. Sci. 16, 9-643 (2015).

17. Glazebrook, J. Contrasting mechanisms of defense against biotrophic and necrotrophic pathogens. Annu. Rev. Phytopathol. 43, 205-227 (2005).

18. Norman-Setterblad, C., Vidal, S. \& Palva, E. T. Interacting signal pathways control defense gene expression in Arabidopsis in response to cell walldegrading enzymes from Enwinia carotovora. Mol. Plant-Microbe Inter. 13, 430-438 (2000)

19. Kariola, T., Palomäki, T. A., Brader, G. \& Palva, E. T. Enwinia carotovora subsp. carotovora and Erwinia-derived elicitors HrpN and PehA trigger distinct but interacting defense responses and cell death in Arabidopsis. Mol. Plant-Microbe Inter. 16, 179-187 (2003)

20. Li, J., Brader, G. \& Palva, E. T. The WRKY70 transcription factor: a node of convergence for jasmonate-mediated and salicylate-mediated signals in plant defense. Plant Cell 16, 319-331 (2004).
21. Denancé, N., Sánchez-Vallet, A., Goffner, D. \& Molina, A. Disease resistance or growth: the role of plant hormones in balancing immune responses and fitness costs. Front. Plant Sci. 4, 155 (2013).

22. Choi, C. et al. Identification of AtWRKY75 as a transcriptional regulator in the defense response to PCC through the screening of Arabidopsis activationtagged lines. Plant Biotechnol. Rep. 8, 183-192 (2014).

23. Zheng, Z., Qamar, S. A., Chen, Z. \& Mengiste, T. Arabidopsis WRKY33 transcription factor is required for resistance to necrotrophic fungal pathogens. Plant J. 48, 592-605 (2006).

24. Birkenbihl, R. P., Diezel, C. \& Somssich, I. E. Arabidopsis WRKY33 is a key transcriptional regulator of hormonal and metabolic responses toward Botrytis cinerea infection. Plant Physiol. 159, 266-285 (2012).

25. Ko, Y. J. et al. Heterologous expression of the Brassica rapa transcription factor BrWRKY7 enhances resistance against bacterial soft rot caused by Pectobacterium carotovorum in Arabidopsis. Plant Biotechnol. Rep. 9, 179-186 (2015).

26. Hwang, B. H. et al. Overexpression of polygalacturonase-inhibiting protein 2 (PGIP2) of Chinese cabbage (Brassica rapa ssp. pekinensis) increased resistance to the bacterial pathogen Pectobacterium carotovorum ssp. carotovorum. Plant Cell Tissue Organ Cult. 103, 293-305 (2010).

27. Jung, Y. J. et al. Overexpression of the pineapple fruit bromelain gene (BAA) in transgenic Chinese cabbage (Brassica rapa) results in enhanced resistance to bacterial soft rot. Electron. J. Biotechnol. 11, 71-79 (2008).

28. Park, Y. H. et al. Over-expression of rice leucine-rich repeat protein results in activation of defense response, thereby enhancing resistance to bacterial soft rot in Chinese cabbage. Plant Cell Rep. 31, 1845-1850 (2012).

29. Wang, $X$. et al. The genome of the mesopolyploid crop species Brassica rapa. Nat. Genet. 43, 1035-1039 (2011).

30. Lu, Y. et al. Construction of one mutant library and research on phenotypic variation of $\mathrm{M}_{2}$ population leaves in Chinese cabbage. Acta Hortic. Sin. $\mathbf{4 1}$ 1609-1619 (2014).

31. Lu, Y. et al. Microspore induced doubled haploids production from ethyl methanesulfonate (EMS) soaked flower buds is an efficient strategy for mutagenesis in Chinese cabbage. Front. Plant Sci. 7, 1780 (2016).

32. Wang, Z., Gerstein, M. \& Snyder, M. RNA-Seq: a revolutionary tool for transcriptomics. Nat. Rev. Genet. 10, 57-63 (2009).

33. Yuan, F., Qu, S. P., Cui, C. S. \& Cao, M. Q. \& Ma, R. C. A new strain of Enwinia carotovora subsp. carotovora isolated from soft-rotted Chinese cabbage. Acta Microbiol. Sin. 44, 136-140 (2004).

34. Zhang, S. H., Yang, Q. \& Ma, R. C. Erwinia carotovora ssp. carotovora infection induced "defense lignin" accumulation and lignin biosynthetic gene expression in Chinese cabbage (Brassica rapa L. ssp. pekinensis). J. Integr. Plant Biol. 49 993-1002 (2007).

35. Trapnell, C. et al. Transcript assembly and quantification by RNA-Seq reveals unannotated transcripts and isoform switching during cell differentiation. Nat. Biotechnol. 28, 511-515 (2010).

36. Livak, K. J. \& Schmittgen, T. D. Analysis of relative gene expression data using real-time quantitative $P C R$ and the $2^{-\Delta C T}$ method. Methods 25, 402-408 (2001).

37. Liao, Y. C., Song, M., Wang, H., Xu, D. H. \& Wang, X. W. Glucosinolate profile and accumulation in Brassica campestris L. ssp. Pekin. Acta Hortic. Sin. 38, 963-969 (2011).

38. He, H. J., Chen, H. \& Schnitzler, W. H. Glucosinolate composition and contents in Brassica vegetables. Acta Hortic. Sin. 35, 192-197 (2002).

39. Johnson, D. B., Moore, W. E. \& Zank, L. C. The spectrophotometric determination of lignin in small wood samples. Tappi 44, 793-798 (1961).

40. Du, F., Ruan, G. \& Liu, H. Analytical methods for tracing plant hormones. Anal. Bioanal. Chem. 403, 55-74 (2012).

41. Ding, $X$. et al. Activation of the indole-3-acetic acid-amido synthetase $\mathrm{GH} 3-8$ suppresses expansin expression and promotes salicylate-and jasmonateindependent basal immunity in rice. Plant Cell 20, 228-240 (2008).

42. Görlach, J. et al. Benzothiadiazole, a novel class of inducers of systemic acquired resistance, activates gene expression and disease resistance in wheat Plant Cell 8, 629-643 (1996).

43. Li, P. Investigations on dominant glucosinolates in rapeseed germplasm collected in china. Sci. Agric. Sin. 32(supplement), 120-123 (1999).

44. Brown, A. F. et al. Glucosinolate profiles in broccoli: variation in levels and implications in breeding for cancer chemoprotection. J. Am. Soc. Hortic. Sci. 127, 807-813 (2002).

45. Peng, $X$. et al. Constitutive expression of rice WRKY30 gene increases the endogenous jasmonic acid accumulation, $P R$ gene expression and resistance to fungal pathogens in rice. Planta 236, 1485-1498 (2012). 
46. Wang, D., Pajerowska-Mukhtar, K., Culler, A. H. \& Dong, X. Salicylic acid inhibits pathogen growth in plants through repression of the auxin signaling pathway. Curr. Biol. 17, 1784-1790 (2007).

47. Mithen, R. Leaf glucosinolate profiles and their relationship to pest and disease resistance in oilseed rape. Euphytica 63, 71-83 (1992).

48. Gigolashvili, T. \& Flügge, U. I. The plastidic bile acid transporter 5 is required for the biosynthesis of methionine-derived glucosinolates in Arabidopsis thaliana. Plant Cell 21, 1813-1829 (2009).

49. Kim, Y. B. et al. MYB transcription factors regulate glucosinolate biosynthesis in different organs of Chinese cabbage (Brassica rapa ssp. pekinensis). Molecules 18, 8682-8695 (2013).

50. Sønderby, I. E., Geu-Flores, F. \& Halkier, B. A. Biosynthesis of glucosinolatesgene discovery and beyond. Trends Plant. Sci. 15, 283-290 (2010).

51. Pfalz, M. et al. Metabolic engineering in Nicotiana benthamiana reveals key enzyme functions in Arabidopsis indole glucosinolate modification. Plant Cell 23, 716-729 (2011).

52. Roux, M. et al. The Arabidopsis leucine-rich repeat receptor-like kinases BAK1/ SERK3 and BKK1/SERK4 are required for innate immunity to hemibiotrophic and biotrophic pathogens. Plant Cell 23, 2440-2455 (2011).

53. Brutus, A., Sicilia, F., Macone, A., Cervone, F. \& De Lorenzo, G. A domain swap approach reveals a role of the plant wall-associated kinase 1 (WAK1) as a receptor of oligogalacturonides. Proc. Natl. Acad. Sci. USA 107, 9452-9457 (2010).

54. Lorenzo, O., Piqueras, R., Sánchez-Serrano, J. J. \& Solano, R. ETHYLENE RESPONSE FACTOR1 integrates signals from ethylene and jasmonate pathways in plant defense. Plant Cell 15, 165-178 (2003).

55. Abe, A. et al. Genome sequencing reveals agronomically important loci in rice using MutMap. Nat. Biotechnol. 30, 174-178 (2012).

56. Barilli, E., Rubiales, D., Amalfitano, C., Evidente, A. \& Prats, E. BTH and BABA induce resistance in pea against rust (Uromyces pisi) involving differential phytoalexin accumulation. Planta 242, 1095-1106 (2015).

57. Lai, Z. et al. Arabidopsis sigma factor binding proteins are activators of the WRKY33 transcription factor in plant defense. Plant Cell 23, 3824-3841 (2011).

58. Panstruga, R., Parker, J. E. \& Schulze-Lefert, P. Snapshot: plant immune response pathways. Cell 136, 978.e1-e3 (2009).

59. Macho, A. P. \& Zipfel, C. Plant PRRs and the activation of innate immune signaling. Mol. Cell 54, 263-272 (2014).

60. Dodds, P. N. \& Rathjen, J. P. Plant immunity: towards an integrated view of plant-pathogen interactions. Nat. Rev. Genet. 11, 539-548 (2010).

61. Miya, A. et al. CERK1, a LysM receptor kinase, is essential for chitin elicitor signaling in Arabidopsis. Proc. Natl Acad. Sci. 104, 19613-19618 (2007)

62. Willmann, R. et al. Arabidopsis lysin-motif proteins LYM1 LYM3 CERK1 mediate bacterial peptidoglycan sensing and immunity to bacterial infection. Proc. Natl Acad. Sci. USA 108, 19824-19829 (2011).

63. Krol, E. et al. Perception of the Arabidopsis danger signal peptide 1 involves the pattern recognition receptor atpepr1 and its close homologue atpepr2. J. Biol. Chem. 285, 13471-13479 (2010).

64. Tang, J. et al. Structural basis for recognition of an endogenous peptide by the plant receptor kinase PEPR1. Cell Res. 25, 110-120 (2015).

65. Gravino, M. et al. Immune responses induced by oligogalacturonides are differentially affected by AvrPto and loss of BAK1/BKK1 and PEPR1/PEPR2. Mol. Plant Pathol. 18, 582-595 (2017).

66. Ferrari, S. et al. Oligogalacturonides: plant damage-associated molecular patterns and regulators of growth and development. Front. Plant Sci. 4, 49 (2013).

67. Kohorn, B. D. Cell wall-associated kinases and pectin perception. J. Exp. Bot. 67 489-494 (2016).

68. Di, C., Zhang, M., Xu, S., Cheng, T. \& An, L. Role of poly-galacturonase inhibiting protein in plant defense. Crit. Rev. Microbiol. 32, 91-100 (2008).

69. De Lorenzo, G. \& Ferrari, S. Polygalacturonase-inhibiting proteins in defense against phytopathogenic fungi. Curr. Opin. Plant. Biol. 5, 295-299 (2002).

70. Galletti, R. et al. The atrbohd-mediated oxidative burst elicited by oligogalacturonides in Arabidopsis is dispensable for the activation of defense responses effective against Botrytis cinerea. Plant Physiol. 148, 1695-1706 (2008).

71. Lai, Z., Wang, F., Zheng, Z., Fan, B. \& Chen, Z. A critical role of autophagy in plant resistance to necrotrophic fungal pathogens. Plant J. 66, 953-968 (2011).
72. Clay, N. K., Adio, A. M., Denoux, C., Jander, G. \& Ausubel, F. M. Glucosinolate metabolites required for an Arabidopsis innate immune response. Science $\mathbf{3 2 3}$ 95-101 (2009).

73. Yan, X. \& Chen, S. Regulation of plant glucosinolate metabolism. Planta 226, 1343-1352 (2007).

74. Schlaeppi, K., Abou-Mansour, E., Buchala, A. \& Mauch, F. Disease resistance of Arabidopsis to Phytophthora brassicae is established by the sequential action of indole glucosinolates and camalexin. Plant J. 62, 840-851 (2010).

75. Kroymann, J. et al. A gene controlling variation in Arabidopsis glucosinolate composition is part of the methionine chain elongation pathway. Plant Physiol. 127, 1077-1088 (2001).

76. Brader, G. et al. Altering glucosinolate profiles modulates disease resistance in plants. Plant J. 46, 758-767 (2006).

77. Hansen, C. H. et al. Cytochrome p450 CYP79F1 from Arabidopsis catalyzes the conversion of dihomomethionine and trihomomethionine to the corresponding aldoximes in the biosynthesis of aliphatic glucosinolates. J. Biol. Chem. 276, 11078-11085 (2001)

78. Bak, S., Tax, F. E., Feldmann, K. A., Galbraith, D. W. \& Feyereisen, R. CYP83B1, a cytochrome P450 at the metabolic branch point in auxin and indole glucosinolate biosynthesis in Arabidopsis. Plant Cell 13, 101-111 (2001).

79. Naur, P. et al. CYP83A1 and CYP83B1, two nonredundant cytochrome P450 enzymes metabolizing oximes in the biosynthesis of glucosinolates in Arabidopsis. Plant Physiol. 133, 63-72 (2003).

80. Kliebenstein, D. J., Lambrix, V. M., Reichelt, M., Gershenzon, J. \& Mitchellolds, T. Gene duplication in the diversification of secondary metabolism: tandem 2oxoglutarate-dependent dioxygenases control glucosinolate biosynthesis in Arabidopsis. Plant Cell 13, 681-693 (2001).

81. Neal, C. S., Fredericks, D. P., Griffiths, C. A. \& Neale, A. D. The characterisation of AOP2: a gene associated with the biosynthesis of aliphatic alkenyl glucosinolates in Arabidopsis thaliana. BMC Plant Biol. 10, 170 (2010).

82. Halkier, B. A. \& Gershenzon, J. Biology and biochemistry of glucosinolates. Annu. Rev. Plant. Biol. 57, 303-333 (2006).

83. Nafisi, M. et al. Arabidopsis cytochrome P450 monooxygenase 71 A13 catalyzes the conversion of indole-3-acetaldoxime in camalexin synthesis. Plant Cell 19, 2039-2052 (2007).

84. Qi, L. et al. Arabidopsis thaliana plants differentially modulate auxin biosynthesis and transport during defense responses to the necrotrophic pathogen Alternaria brassicicola. New Phytol. 195, 872-882 (2012).

85. Ludwig-Müller, J. Auxin conjugates: their role for plant development and in the evolution of land plants. J. Exp. Bot. 62, 1757-1773 (2011).

86. Jagadeeswaran, G. et al. Arabidopsis GH3-LIKE DEFENSE GENE 1 is required for accumulation of salicylic acid, activation of defense responses and resistance to Pseudomonas syringae. Plant J. 51, 234-246 (2007).

87. $\mathrm{Fu}, \mathrm{J}$. et al. Manipulating broad-spectrum disease resistance by suppressing pathogen-induced auxin accumulation in rice. Plant Physiol. 155, 589-602 (2011).

88. Takase, T. et al. ydk1-D, an auxin-responsive GH3 mutant that is involved in hypocotyl and root elongation. Plant J. 37, 471-483 (2004).

89. Gfeller, A., Liechti, R. \& Farmer, E. E. Arabidopsis jasmonate signaling pathway. Sci. Signal. 3, cm4 (2010).

90. Han, G. Z. Evolution of jasmonate biosynthesis and signaling mechanisms. J. Exp. Bot. 68, 1323-1331 (2016).

91. Broekaert, W. F., Delauré, S. L., De Bolle, M. F. \& Cammue, B. P. The role of ethylene in host-pathogen interactions. Annu. Rev. Phytopathol. 44, 393-416 (2006).

92. Thomma, B. P., Penninckx, I. A., Cammue, B. P. \& Broekaert, W. F. The complexity of disease signaling in Arabidopsis. Curr. Opin. Immunol. 13, 63-68 (2001).

93. Dixon, R. A. Natural products and plant disease resistance. Nature 411, 843-847 (2001).

94. Egea, C., Ahmed, A. S., Candela, M. \& Candela, M. E. Elicitation of peroxidase activity and lignin biosynthesis in pepper suspension cells by Phytophthora capsici. J. Plant. Physiol. 158, 151-158 (2001). 